\title{
X-ray view of IC 348 in the light of an updated cluster census ${ }^{\star}$
}

\author{
B. Stelzer ${ }^{1}$, T. Preibisch ${ }^{2}$, F. Alexander ${ }^{2}$, P. Mucciarelli ${ }^{2}$, E. Flaccomio ${ }^{1}$, G. Micela ${ }^{1}$, and S. Sciortino ${ }^{1}$ \\ 1 INAF - Osservatorio Astronomico di Palermo, Piazza del Parlamento 1, 90134 Palermo, Italy \\ e-mail: stelzer@astropa.inaf.it \\ 2 Universitäts-Sternwarte München, Ludwig-Maximilians-Universität, Scheinerstr. 1, 81679 München, Germany
}

Received 17 September 2011 / Accepted 8 November 2011

\begin{abstract}
Context. IC 348 is a nearby $(\approx 310 \mathrm{pc})$, young $(\sim 2-3 \mathrm{Myr})$ open cluster with $>300$ members identified from optical and infrared observations. It comprises young stellar objects in various evolutionary phases from protostars over disk-bearing to diskless premain sequence stars. This gives us the opportunity to study evolutionary effects in the high-energy emissions in a homogeneous environment.

Aims. We study the properties of the coronae of the young low-mass stars in IC 348 combining X-ray and optical/infrared data. In particular, we intend to shed light on the dependence of X-ray luminosity and spectral hardness on evolutionary stage and on stellar parameters such as mass, effective temperature, and bolometric luminosity.

Methods. The four existing Chandra observations of IC 348 were merged, thus providing a deeper and spatially more complete X-ray view than previous X-ray studies of the cluster. We have compiled a comprehensive catalog of IC 348 members taking into account recent updates to the cluster census. Our data collection comprises fundamental stellar parameters, infrared excess indicating the presence of disks, $\mathrm{H} \alpha$ emission as a tracer of chromospheric emission or accretion, and mass accretion rates.

Results. We have detected 290 X-ray sources in four merged Chandra exposures, of which 187 are associated with known cluster members, which corresponds to a detection rate of $\sim 60 \%$ for the cluster members of IC 348 that are identified in optical/infrared studies. According to the most recent spectral classification of IC 348 members, only four of the X-ray sources are brown dwarfs (spectral type M6 and later). The detection rate is highest for diskless Class III stars and increases with stellar mass. This may be explained with higher X-ray luminosities for higher mass and later evolutionary stage that is evident in the X-ray luminosity functions. In particular, we find that for the lowest examined masses $\left(0.1-0.25 M_{\odot}\right)$ there is a difference between the X-ray luminosity functions of accreting and non-accreting stars (classified on the basis of their $\mathrm{H} \alpha$ emission strength) as well as those of disk-bearing and diskless stars (classified on the basis of the slope of the spectral energy distribution). These differences disappear for higher masses. This is related to our finding that the $L_{\mathrm{x}} / L_{\mathrm{bol}}$ ratio is non-constant across the mass/luminosity sequence of IC 348 with a decrease toward lower luminosity stars. Our analysis of an analogous stellar sample in the Orion Nebula Cluster suggests that the decline of $L_{\mathrm{x}} / L_{\mathrm{bol}}$ for young stars at the low-mass end of the stellar sequence is likely universal.
\end{abstract}

Key words. stars: pre-main sequence - X-rays: stars - stars: activity - stars: coronae

\section{Introduction}

The open cluster IC 348 is arguably the best investigated rich (>100 members), and very young ( $2-3$ Myr old) stellar cluster within $\sim 300 \mathrm{pc}$ from the Sun. IC 348 is associated to the Perseus molecular cloud complex at a distance of about $310 \mathrm{pc}$ (Herbig 1998) and located just to the south of the optically bright star $o$ Per (B1 III). The cluster members spread over about $15^{\prime} \times$ $15^{\prime}$ on the sky. The optically brightest and most massive cluster member is the $\mathrm{B} 5 \mathrm{~V}$ star $\mathrm{BD}+31^{\circ} 643$.

In a large number of observational studies, more than 300 individual cluster members have been identified and well characterized so far. The pioneering study of Herbig (1954) discovered $16 \mathrm{H} \alpha$ emitting stars in IC 348. Later, Herbig (1998) identified about 100 members by optical spectroscopy and photometry as well as deep $\mathrm{H} \alpha$ imaging. The near-infrared photometric survey of Lada \& Lada (1995), and later, deeper infrared (IR) imaging studies were used to construct the luminosity function of the cluster and provided information on the stellar and substellar

* Full Tables 3 and 7 are available at the CDS via anonymous ftp to cdsarc.u-strasbg.fr $(130.79 .128 .5)$ or via

http://cdsarc.u-strasbg.fr/viz-bin/qcat?]/A+A/537/A135 mass function (Lada et al. 1998; Muench et al. 2003; Preibisch 2003).

By means of optical and IR spectroscopy and photometry, the investigations of Luhman et al. (1998); Luhman (1999); Luhman et al. (2003, 2005a) progressively increased the completness of the census of the young stellar population in IC 348 . These studies yielded a sample of 302 well characterized members, for most of which individual stellar masses and ages can be reliably estimated. Although the cluster contains a few embedded stars, this extinction-limited $\left(A_{\mathrm{V}} \leq 4 \mathrm{mag}\right)$ sample of members is nearly complete down to masses of $M \geq 0.03 M_{\odot}$; it also contains 23 spectroscopically identified young brown dwarfs (BDs), with spectral types as late as M9 and estimated masses down to $0.015 M_{\odot}$. Recently, Burgess et al. (2009) discovered a young T-dwarf candidate in IC 348. If its status as a cluster member is confirmed, the derived spectral type of $\sim \mathrm{T} 6$ suggests a mass of only a few Jupiter masses.

IC 348 was also extensively studied in the near- and midIR with the Spitzer Space Telescope. Lada et al. (2006) constructed optical/IR spectral energy distributions (SEDs) for all cluster members and investigated both the frequency and nature of the circumstellar disk population in the cluster. Muench et al. (2007) performed a Spitzer census of IC 348 and found that 
about $50 \%$ of the known cluster members possess circumstellar disks. Currie \& Kenyon (2009) presented new, deep MIPS photometry of IC 348 that allowed a detailed characterization of the circumstellar disks around the known cluster members. Finally, millimetric observations directly revealed the presence and mass of 10 disks around IC 348 members (Lee et al. 2011). Dahm (2008) performed a spectroscopic investigation of accretion diagnostics for 40 near solar-mass members of IC 348 and derived accretion luminosities and rates for 14 of these stars.

Based on all these studies, the basic properties of IC 348 can be summarized as follows: The mean age of the cluster members of $\sim 2-3 \mathrm{Myr}$ is very interesting because it corresponds to the time where the structure of the disks of most young stellar objects changes from primordial, rather massive accretion disks to transitional and debris disks, and the point in time when planetary formation is thought to occur. Furthermore, it offers interesting opportunities for comparisons to younger clusters like the Orion Nebula Cluster (1-2 Myr; Hillenbrand 1997) or the $\rho$ Oph cluster ( $\leq 1$ Myr; Luhman \& Rieke 1999), and older clusters and associations such as Upper Scorpius ( $5 \mathrm{Myr}$; de Geus et al. 1989).

The extinction of the cluster members ranges from $A_{V} \sim$ $1 \mathrm{mag}$ up to $A_{V} \gtrsim 10 \mathrm{mag}$, with a mean value of $\sim 3.5 \mathrm{mag}$. The majority of the cluster members is in the T Tauri stage of premain sequence evolution, and more than 70 stars can be classified as "classical T Tauri stars" (CTTS) on the basis of their $\mathrm{H} \alpha$ emission. In the central parts of the cluster the active starformation phase seems to be finished, and the cluster population represents the outcome of a recent star-formation event. About $10^{\prime}$ to the southwest of the cluster center, however, a dense cloud core is found that contains several deeply embedded IR sources with extinctions exceeding $\sim 20 \mathrm{mag}$ in $A_{V}$ as well as the very young molecular hydrogen jet HH 211 (McCaughrean et al. 1994) and the IC 348 MMS outflow (Eislöffel et al. 2003). The star formation process is still ongoing in this region.

IC 348 is also well studied in the X-ray regime. The first X-ray observations of IC 348 by Preibisch et al. (1996) were performed with the ROSAT observatory and led to the discovery of 116 X-ray sources. In September 2000, a deep X-ray image of IC 348 was obtained with the Advanced CCD Imaging Spectrometer (ACIS) on board the Chandra X-Ray Observatory and led to the detection of $215 \mathrm{X}$-ray sources in the $17^{\prime} \times 17^{\prime}$ field-of-view. These Chandra observations were analyzed by Preibisch \& Zinnecker (2001, PZ01 hereafter) and Preibisch \& Zinnecker (2002, PZ02 hereafter). In February 2003, IC 348 was observed with XMM-Newton (Preibisch \& Zinnecker 2004). The $X M M-N e w t o n$ observation was strongly affected by solar particle flares and consequently the sensitivity was significantly reduced. Therefore, only a small number of new X-ray sources were detected in the field previously observed with Chandra. Two more recent Chandra observations centered on the southwestern corner of IC 348 were analyzed by Forbrich et al. (2011). They detected about one-third of the known cluster members in this area.

While the analysis of these X-ray studies yielded numerous interesting results about the X-ray properties of the young stars in IC 348, most of the above mentioned optical/IR studies of the cluster were not yet available at the time. (The recent Chandra study of the fields in the southwest of the cluster by Forbrich et al. 2011 focused on a comparison of radio and X-ray emission of YSOs without detailed consideration of the stellar properties.) The very substantial increase of optical/IR information about the cluster population during the last $\sim$ five years clearly warrants a repeated effort to study the relations between X-ray properties and stellar parameters. Another important aspect in this context is the availability of three new deep Chandra observations of IC 348, obtained in 2008 and that spatially overlap with the original field. The resulting Chandra "mosaic" comprises more than $90 \%$ of the cluster population; see Sect. 4.1 for more details.

During the last few years, several very deep and extensive $\mathrm{X}$-ray studies of other low-mass star-forming regions have been performed, which provide a good basis for comparisons of the $\mathrm{X}$-ray properties of young stars at different ages and in different environments. The first of these studies was the Chandra Orion Ultradeep Project (COUP), a 10-day long observation of the Orion Nebula Cluster (ONC) with Chandra/ACIS (see Getman et al. 2005), the deepest and longest X-ray observation ever made of a young stellar cluster. A detailed investigation of the relation between the optical and X-ray properties of the $\sim 600$ X-ray detected optically visible and well characterized cluster members was presented in Preibisch et al. (2005a) and Stelzer et al. (2005). The XMM-Newton Extended Survey of the Taurus Molecular Cloud (XEST) covered the densest stellar populations in a 5 square degree region of the Taurus Molecular Cloud (see Güdel et al. 2007) and provided X-ray data on 110 optically well characterized young stars.

The analysis presented in this paper uses all available Chandra observations of IC 348 to increase the spatial coverage of the cluster and the sensitivity in the region where the pointings overlap. The X-ray source list is cross-correlated with an updated membership catalog that we have compiled from the literature. This yields stellar parameters, disk and accretion indicators and rotation rates for the majority of X-ray emitting YSOs in IC 348. We examine the dependence of X-ray emission level on these parameters and compare the results to X-ray studies of other nearby low-mass star forming regions such as Orion and Taurus with the aim of understanding the origin of activity in pre-main sequence stars.

\section{The catalog of IC 348 members}

\subsection{The data base}

Since the studies by PZ01, the membership list of IC 348 has changed significantly with more than 130 new members confirmed by signatures of youth in optical low-resolution spectra and their position in the HR diagram above the $10 \mathrm{Myr}$ isochrone (Luhman et al. 2003, henceforth LSM 03). LSM 03 considered membership complete in the central $16^{\prime} \times 14^{\prime}$ for $M>0.03 M_{\odot}$ and $A_{\mathrm{V}}<4$ mag. However, additional members have been identified on the basis of IR spectroscopy (Luhman et al. 2005b, henceforth LLM 05), and Spitzer mid-IR photometry (Muench et al. 2007), of which about a dozen in the LSM 03 completeness area. Several objects that had late-M spectral types estimated previously from HST near-IR photometry (Najita et al. 2000) have been rejected by LSM03 as cluster members on the basis of their optical spectra.

Spitzer photometry from IRAC and MIPS for the members from LSM 03 was discussed by Lada et al. (2006). They examined the slope of the SED between $3.6 \ldots 8 \mu \mathrm{m}$ for 263 objects with IRAC photometry in all bands, and distinguished protostars, disk-bearing stars and disk-less stars in four categories that we describe in Sect. 2.2.

The present paper deals with the more than $90 \%$ of known IC 348 members that are within the field of the presently available Chandra observations. The catalog of optical/IR cluster members consists of the objects from Table 2 in LSM03, Table 2 in LLM05 and Table 1 in Muench et al. (2007). These catalogs provide spectral types, effective temperatures $\left(T_{\text {eff }}\right)$, extinctions 
Table 1. Definition of YSO class.

\begin{tabular}{lc}
\hline \hline Class & $\alpha_{3-8 \mu \mathrm{m}^{*}}$ \\
\hline I & $>-0.5$ \\
II & $-0.5 \ldots . .-1.8$ \\
II/III & $-1.8 \ldots-2.56$ \\
III & $<-2.56$ \\
\hline
\end{tabular}

Notes. ${ }^{(*)}$ Slope of the SED from 3-8 $\mu$ m (adopted from Lada et al. 2006; and Muench et al. 2007).

( $A_{\mathrm{J}}$ or $A_{\mathrm{V}}$ ), bolometric luminosities $\left(L_{\mathrm{bol}}\right)$, and optical/nearIR photometry. For the objects from Luhman's papers Spitzer photometry is extracted from Lada et al. (2006). Accretion rates are collected from Dahm (2008); Muzerolle et al. (2003); Mohanty et al. (2005), and $\mathrm{H} \alpha$ equivalent widths are those listed in LSM03.

\subsection{Derived properties}

We have complemented the data base described above by several properties derived from the literature data.

First, YSOs are classified in two different ways, (i) on the basis of the IR slope of their SED, and (ii) on the basis of their $\mathrm{H} \alpha$ equivalent width.

For the SED classification the slopes $\alpha_{3-8 \mu \mathrm{m}}$ derived by Lada et al. (2006) and Muench et al. (2007) are used together with the boundaries defined by Lada et al. (2006) that separate YSOs in different evolutionary phases (see Table 1). The four groups represent Class I objects (protostars), Class II (stars with thick disks), Class II/III (stars with "anemic" disks), and Class III (disk-less stars). The group termed "anemic" disks fills the gap between disk-bearing and disk-less stars in Spitzer/IRAC color-color diagrams. This group shows a very small spread of [3.6]-[8.0] color which forms the lower envelope to thick-disk stars.

The distinction of classical and weak-line T Tauri stars (henceforth cTTS and wTTS) on the basis of the strength of $\mathrm{H} \alpha$ emission must consider the fact that the $\mathrm{H} \alpha$ equivalent width $\left(W_{\mathrm{H} \alpha}\right)$ is not equivalent to the line flux as a result of its dependence on the continuum, which declines toward later spectral types. Therefore, when using $W_{\mathrm{H} \alpha}$ as an accretion indicator, the threshold between cTTS and wTTS must be set at successively higher values for stars of later spectral types. We used thresholds of 3, 10, and $20 \AA$ for stars earlier than K5, between K6 and M3, and later than M3, respectively. This is similar to the criteria introduced by White \& Basri (2003), who included an additional group with $W_{\mathrm{H} \alpha}>40 \AA$ for stars later than M6. The omission of this last step is justified by the separation of Class II and Class III sources in Fig. 1.

A comparison of the two ways used to classify YSO evolution gives clues to the connection between the dissipation of dust and gas during the pre-main sequence. Lada et al. (2006) found that in IC 348 there is a close relation between $\mathrm{H} \alpha$ equivalent width and IR excess. We confirm this in Fig. 1 using a more sophisticated division between accretors and non-accretors that depends on spectral type. The majority of Class II sources are classified as accretors on the basis of their $\mathrm{H} \alpha$ emission. Only one Class III source has an $\mathrm{H} \alpha$ equivalent width in the range typical for accretors, and only 6 out of 28 Class II/III sources. However, next to the majority of Class II/III sources, several Class II sources are classified as "weak-lined" stars according to our criterion, i.e. they have disks but are not considered to

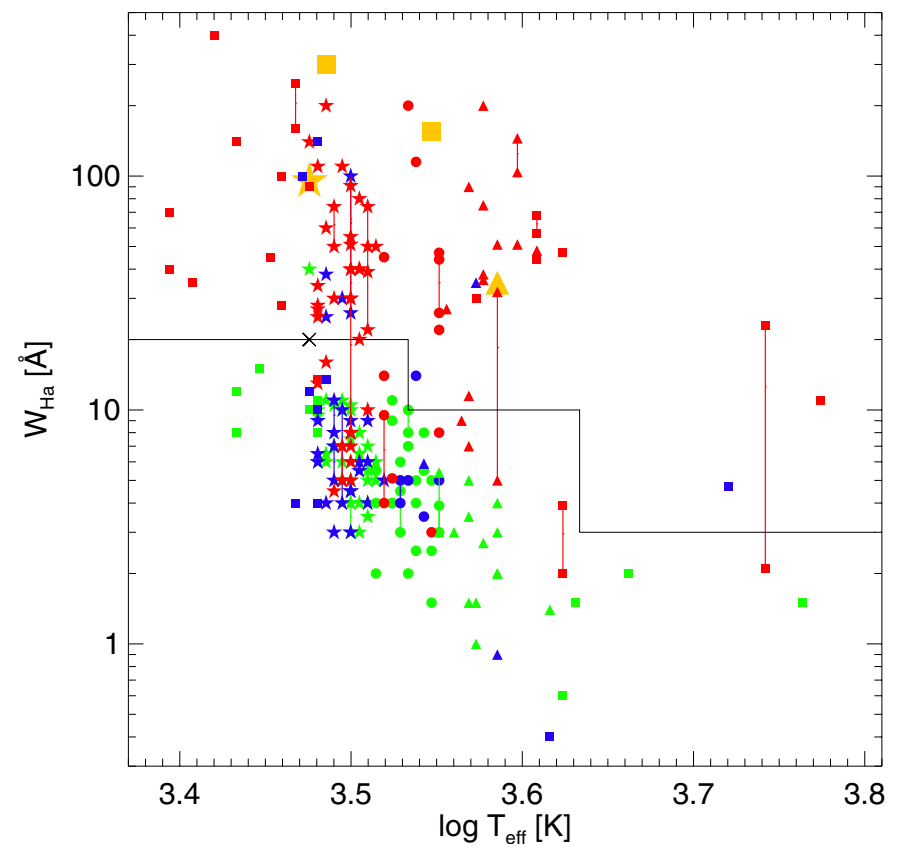

Fig. 1. Equivalent width of $\mathrm{H} \alpha$ vs. effective temperature. The color-codes refer to the Spitzer YSO classes: Class III (green; light gray in b/w print), Class II/III (blue; dark gray in b/w print), Class II (red; medium gray in $\mathrm{b} / \mathrm{w}$ print) and Class I (yellow and larger). Different mass ranges are symbolized with different plotting symbols: $0.1-0.25 M_{\odot}$ (star symbols), 0.25-0.6 $M_{\odot}$ (circles), 0.6-1.2 $M_{\odot}$ (triangles) and all others (squares). Vertical lines connect different epochs of $W_{\mathrm{H} \alpha}$ measurements for individual stars. The black line separates accreting cTTS (top) from non-accreting wTTS (bottom). The cross on the black line denotes spectral type M6. For objects with $T_{\text {eff }}$ smaller than this value White \& Basri (2003) have placed the dividing line between cTTS and wTTS at $40 \AA$. However, our boundary of $20 \AA$ agrees better with the separation of Class II and Class III sources.

be actively accreting any more presumably because the gas has dispersed. These objects are likely the more evolved among the Class II and Class II/III stars. Another possible explanation for the presence of Class II sources below the cTTS cutoff line is variable accretion that would allow for some disk-bearing Class II and Class II/III objects to appear temporarily as wTTS. Stars with more than one $\mathrm{H} \alpha$ measurement (connected by vertical lines in Fig. 1) show indeed that $\mathrm{H} \alpha$ emission is strongly variable, and the equivalent widths measured at different epochs for some Class II objects do cross the boundary between cTTS and wTTS. For those stars we have used the highest value of $W_{\mathrm{H} \alpha}$ as criterion for their cTTS/wTTS classification.

It can also be seen from Fig. 1 that a few sources classified as Class I have not only a determined spectral type but also detected $\mathrm{H} \alpha$ emission, both unexpected for true protostars that ought to be invisible in the optical due to high extinction of their envelopes. In total, among the 15 Class I sources known in IC 348, a spectral type has been detected for ten of them and five have a measurement for $\mathrm{H} \alpha$ emission. The $\mathrm{H} \alpha$ equivalent widths of these stars are all above our "accretor threshold". These objects are probably no genuine protostars but T Tauri stars with massive disks that are responsible for their shallow slope in the mid-IR. Indeed, Muench et al. (2007) state that the distinction between highly flared Class II disks and disk/envelope systems may require observations at wavelengths longward of $10 \mu \mathrm{m}$. Such measurements are not available for the majority of our sample stars, and 


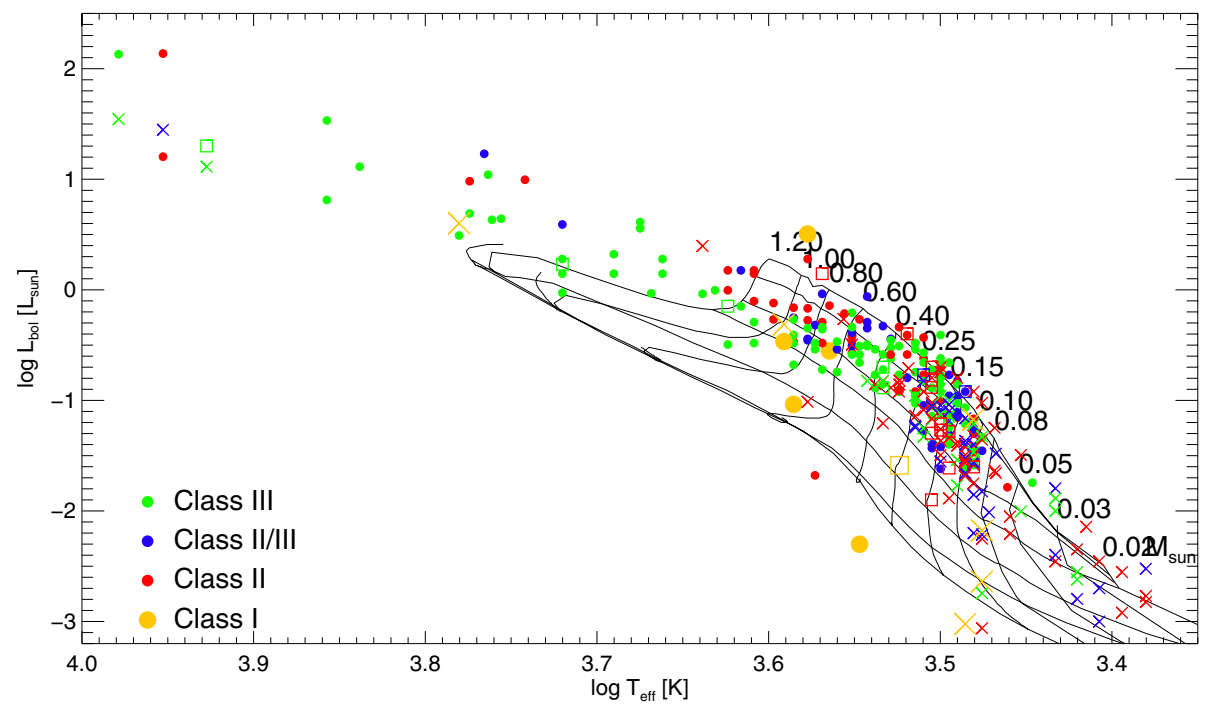

Fig. 2. HR diagram for IC 348 compared to evolutionary tracks of Baraffe et al. (1998) and Chabrier et al. (2000). Isochrones are shown for $1,2,5,10,30,100,1000 \mathrm{Myr}$. The tracks are labeled in units of solar mass. X-ray detections are represented by filled circles and nondetections as crosses. IC 348 members outside the Chandra fields are shown as open squares. The objects are color-coded according to their YSO class (see definition in Sect. 2.2).

Table 2. Chandra observation log.

\begin{tabular}{|c|c|c|c|c|c|c|c|c|}
\hline Obs. Id. & \multicolumn{2}{|c|}{ Date start [UT] } & \multicolumn{2}{|c|}{ Date end [UT] } & Exposure time & Level 2 events & $\alpha_{2000}[\mathrm{~h}: \mathrm{m}: \mathrm{s}]$ & $\delta_{2000}\left[{ }^{\circ}:^{\prime}::^{\prime \prime}\right]$ \\
\hline 606 & $2000-09-21$ & $19: 58: 42$ & $2009-09-22$ & $11: 09: 29$ & $52960 \mathrm{~s}$ & 347817 & $03: 44: 30.00$ & $+32: 08: 00.0$ \\
\hline 8584 & $2008-03-15$ & 09:02:20 & 2008-03-15 & $23: 33: 38$ & $50140 \mathrm{~s}$ & 582252 & 03:44:13.20 & $+31: 06: 00.0$ \\
\hline 8933 & 2008-03-18 & 17:35:07 & 2008-03-19 & $15: 12: 52$ & $40120 \mathrm{~s}$ & 294144 & $03: 43: 59.90$ & $+31: 58: 21.7$ \\
\hline 8944 & $2008-03-13$ & $17: 53: 02$ & 2008-03-14 & $05: 25: 12$ & $38640 \mathrm{~s}$ & 300328 & 03:43:59.90 & $+31: 58: 21.7$ \\
\hline
\end{tabular}

in any case they are not taken into account in the definition of YSO classes as described in Table 1.

We sought to determine masses for all stars with tabulated $L_{\mathrm{bol}}$ and $T_{\text {eff }}$ from an interpolation of evolutionary models. Different sets of models are known to be inconsistent between each other. Luhman et al. (2003) have shown that the Baraffe et al. (1998) and Chabrier et al. (2000) models yield the best description for the low-mass stars in IC 348. These are also the only models that include very low mass stars $\left(<0.1 M_{\odot}\right)$ and extend into the BD regime. The HR diagram for IC 348 is shown in Fig. 2 where we anticipate the result from the cross-correlation with X-ray sources (see Sect. 3). Our choice of models is made at the expense of an analysis of stars with $M>1.2 M_{\odot}$. However, as can be seen from Fig. 2, the vast majority of IC 348 members have lower mass. No mass could be determined for a few stars below the zero-age main-sequence. These are probably stars with edge-on disks seen in scattered light, consistent with their classification as YSOs with circumstellar matter (either Class II or Class I).

\section{X-ray data analysis}

IC 348 was observed in September 2000 (ObsID 606, PI: Th. Preibisch) and three times in March 2008 (ObsIDs 8584 PI: N. Calvet, 8933 and 8944 PI: S. Wolk) with the imaging array of the Chandra Advanced CCD Imaging Spectrometer (ACIS-I), which provides a field of view of $17^{\prime} \times 17^{\prime}$ on the sky. The aimpoint of observation 8584 was offset to the southwest with respect to pointing 606 and observations 8933 and 8944 were offset even more to the southwest; see Fig. 3 for a graphical representation of the Chandra observations. The total net exposure time of all four observations was $182860 \mathrm{~s}(50.79 \mathrm{~h})$. Table 2 contains detailed information on the four observations. The roll angles (i.e. the orientation of the detector on the sky) are equal $\left(288-289^{\circ}\right)$ for the three observations from 2008 . For observation 606 the roll angle was $109.6^{\circ}$ and hence the detector was rotated $180^{\circ}$. Therefore sources are only at the same detector position for observations 8933 and 8944 . Two of the CCDs of the ACIS-S spectroscopic array were also turned on during the observations. However, because the PSF at the large offaxis angles at these detectors is strongly degraded, their pointsource sensitivity is reduced; analysis was performed without using the data from ACIS-S. The basic data products of our observation are the four Level 2 processed event lists provided by the pipeline processing at the Chandra X-ray Center, which list the arrival time, location on the detector, and energy for each of the 1524541 detected X-ray photons. We combined the four pointings with the merge_all script, a Chandra contributed software that makes use of standard $C I A O^{1}$ tools. The merged events file is shown in Fig. 3. Overlaid are crosses at the positions of the IC 348 members showing the spatial distribution of the YSOs.

\subsection{Source detection and X-ray source catalog}

The source detection was carried out in a two-step process. The first detection step was performed in a fairly aggressive manner to find even the weakest possible sources, deliberately accepting some degree of false detections. In the second step, this list of potential sources was then cleaned from spurious detections by a detailed individual analysis. We employed the WAVDETECT algorithm (a CIAO mexican-hat wavelet source detection tool; Freeman et al. 2002) for locating X-ray sources in our merged image, and used a detection threshold of $10^{-5}$ and wavelet scales between 1 and 16 pixels. The result was a list of 372 prospective sources. Three sources were added, which have been detected with WAVDETECT in an XMM-Newton observation of IC 348. Another 17 possible sources were added, which were identified by cross-correlating UKIRT observations with the Chandra image by eye. These 20 sources were first added as potential sources to the sourcelist for ACIS Extract. ACIS

\footnotetext{
1 Chandra Interactive Analysis of Observations, version 4.2: http: //cxc.harvard.edu/ciao/index.html
} 


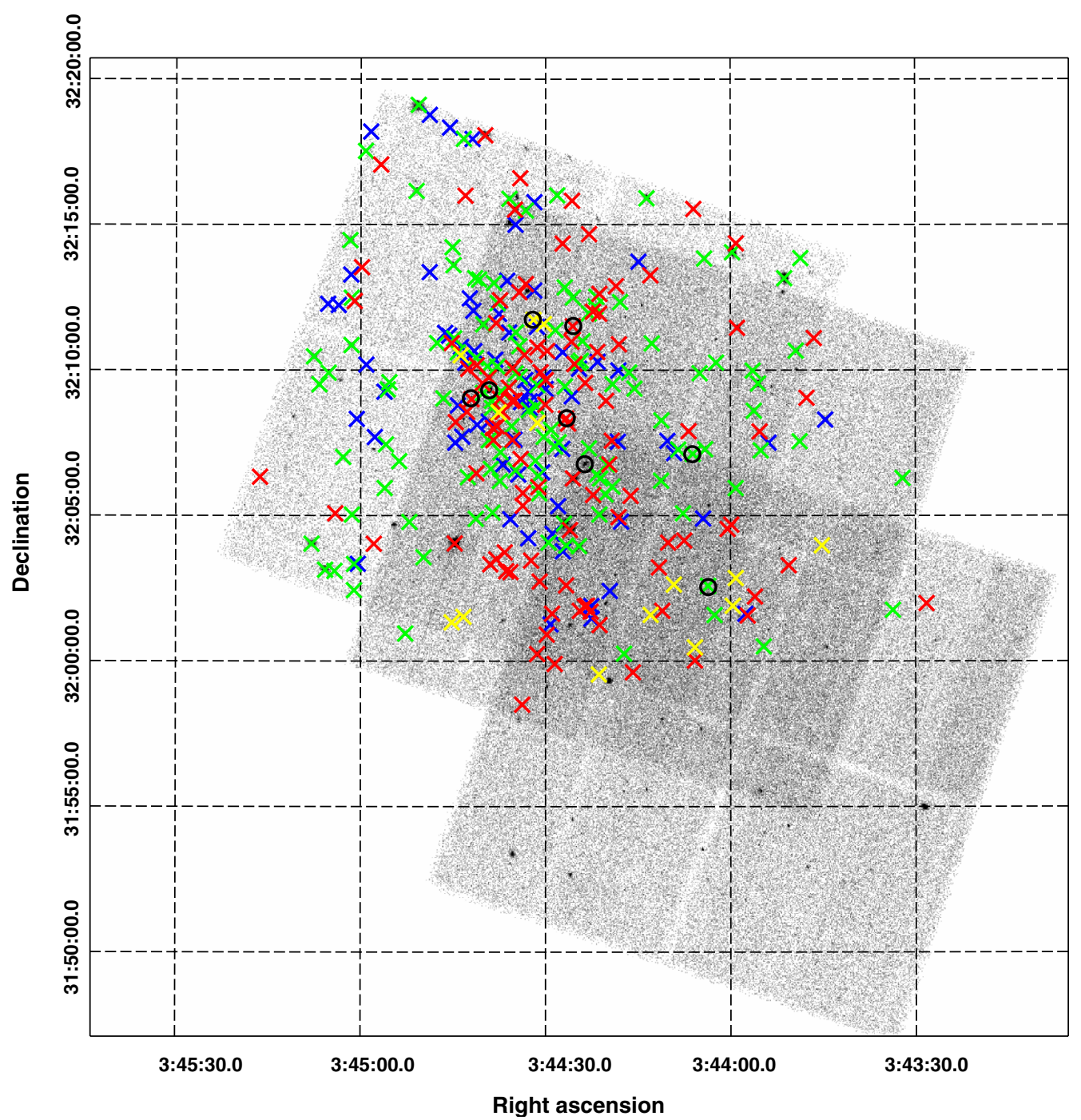

Fig. 3. Merged Chandra/ACIS-I image created from the four observations listed in Table 2. IC 348 members in different YSO phases are color-coded as in Fig. 1. Cluster members without known YSO class are shown as black circles; many of these are secondary components in binaries.

Extract then constructs an extraction region at the position of these sources and estimates the probability for being a source. The result was a final catalog with 393 potential X-ray sources. To clean this catalog from spurious sources, we performed a detailed analysis of each individual candidate source with the ACIS Extract (AE hereafter) software package ${ }^{2}$ (Broos et al. 2010). A full description of the procedures used in AE can be found in Getman et al. (2005), Townsley et al. (2003), and Broos et al. (2007). The following three steps were performed by AE to prune our input catalog from spurious detections (including afterglows):

1. Extraction regions were defined such as to include $90 \%$ of the source photons in the PSF (or less for other nearby sources), and source events were extracted from this region. Ancillary Response Functions (ARFs) and Response Matrix Functions were constructed for each source, and the ARFs were corrected for the light missed by the finite extraction regions.

2. Local background events were extracted after masking all the sources in the catalog.

\footnotetext{
2 http://www.astro.psu.edu/xray/docs/TARA/ ae_users_guide.html
}

3. The Poisson probability $\left(P_{\mathrm{B}}\right)$ associated with the "null hypothesis", i.e. that no source exists and the extracted events are solely caused by Poisson fluctuations in the local background, was computed for each source.

After six iterations of this pruning procedure, our final catalog consisted of 290 X-ray sources, for which the probability of being a background fluctuation is less than $0.1 \%$. For 287 sources of those the probability is less than $0.01 \%$ and 266 sorces have a very low probability of $10^{-5}$. Some of the basic source properties derived by the AE software, such as the net (i.e., backgroundsubtracted) counts in various energy bands, the median photon energy $\left(E_{\text {med }}\right)$, statistical test for variability, and a measure of the incident photon flux, are reported in Table 7. Sources are sorted by increasing right ascension and identified by their sequence number (Col. 1) or their IAU designation (Col. 2).

\subsection{Calculation of $X$-ray fluxes}

An accurate determination of the intrinsic X-ray source luminosities requires good knowledge of the X-ray spectrum. However, for the majority of the X-ray sources the number of detected photons is too low (less than 100 net counts) for a detailed spectral analysis. 
An estimate of the intrinsic, i.e. extinction corrected, X-ray luminosity for sources that are too weak for a detailed spectral analysis can be obtained with the XPHOT software $^{3}$, developed by Getman et al. (2010). XPHOT is based on a non-parametric method for the calculation of fluxes and absorbing X-ray column densities of weak X-ray sources. Our procedure is based on a grid of simulated X-ray spectra with the following parameters: two temperatures, the emission measure ratio of the two spectral components, and the absorbing column. The X-ray extinction and intrinsic flux of a given X-ray source are estimated from a sophisticated comparison scheme between the observed median energy and the observed source flux and analogous values obtained from the simulated spectra. This method requires at least four net counts per source (to determine a meaningful value for the median energy) and can thus be applied to 250 of our 290 sources.

An estimate of the observed (i.e. not the intrinsic) X-ray flux is also computed by AE. This quantity, called FLUX2 and given in units of photons $/ \mathrm{s} / \mathrm{cm}^{2}$, is calculated from the number of detected photons and using a mean value of the instrumental effective area (through the ARF) over energy. It should be noted that the FLUX2 values suffer from a systematic uncertainty with respect to the true incident flux, because the use of a mean ARF is only correct in the hypothetical case of a flat incident spectrum, an assumption that is probably not fulfilled. Nevertheless, the FLUX2 represents the best estimate for the photon flux that can be obtained for comparatively weak sources. The product of $F L U X 2$ and $E_{\text {med }}$ provides an estimate for the energy flux of the source. When compared to the fluxes derived with XPHOT a fairly good linear correlation is found but the product $F L U X 2 \cdot E_{\text {med }}$ results in systematically higher values. We calibrated the FLUX2 values using the correlation with the XPHOT fluxes to obtain source fluxes for the 40 sources that are too faint for XPHOT.

In this way, the X-ray flux of all 290 detected sources has been determined in a consistent way. We cross-correlated the X-ray source list with the catalog of IC 348 members from Sect. 2 using a match radius of $1.5^{\prime \prime}$. Larger cross-correlation radii did not increase the number of matches substantially, e.g. a radius of 3.0" yields only four additional identifications, an increase of the X-ray sample for IC 348 members of about $2 \%$. We are, therefore, confident that nearly all X-ray emitting IC 348 members have been recognized by our procedure. The flux of all $\mathrm{X}$-ray detected IC 348 members is given in Table 3. A distance of $310 \mathrm{pc}$ (Herbig 1998) is assumed for conversion of the flux to luminosity. The resulting intrinsic X-ray luminosities range from $10^{28.38}$ to $10^{31.93} \mathrm{erg} \mathrm{s}^{-1}$.

\subsection{Determination of upper limits}

For those 129 stars from LSM03, LLM05 and Muench et al. (2007), which had no counterpart in our X-ray source catalog, upper limits had to be determined. We did this using again the AE software package. Extraction regions were defined the same way as the $90 \%$ contours of the local PSF to determine the number of counts in the target aperture and an estimate of the local background. To calculate upper limits for the received source counts from AE, we used the program bayes.f (Kraft et al. 1991). This program performs the calculation of the upper limit on a Poisson process with background, incorporating the effects of uncertainty on the background and signal acceptance. Upper limits for the number of source counts were determined for a

\footnotetext{
${ }^{3}$ http://www . astro.psu.edu/users/gkosta/XPHOT/
}

Table 3. X-ray fluxes and upper limits.

\begin{tabular}{|c|c|c|c|}
\hline Chandra ID & $\mathrm{ID}^{a}$ & $\begin{array}{c}\log f_{\mathrm{x}} \\
{\left[\mathrm{erg} / \mathrm{cm}^{2} / \mathrm{s}\right]}\end{array}$ & Flag $^{b}$ \\
\hline & 1 & $<-14.34$ & \\
\hline $034435.35+321004.5$ & 2 & -12.32 & \\
\hline $034450.64+321906.5$ & 3 & -12.10 & \\
\hline $034431.21+320622.1$ & 4 & -13.71 & \\
\hline $034426.03+320430.4$ & 5 & -12.34 & \\
\hline \multirow[t]{3}{*}{$034436.93+320645.4$} & 6 & -12.09 & \\
\hline & 7 & $<-14.35$ & \\
\hline & 8 & $<-14.07$ & \\
\hline $034439.15+320918.1$ & 9 & -12.25 & \\
\hline $034424.66+321014.8$ & 10 & -14.28 & \\
\hline \multirow[t]{3}{*}{$034507.96+320401.8$} & 11 & -12.32 & \\
\hline & 12 & $<-14.78$ & \\
\hline & 12 & $<-14.63$ & \\
\hline $034359.67+320154.1$ & 13 & -12.53 & \\
\hline
\end{tabular}

Notes. ${ }^{(a)}$ Identifier from LSM03, LLM05 or Muench et al. (2007) for all IC 348 members observed with Chandra. ${ }^{(b)}$ Flag is set if the flux was obtained from $\mathrm{AE}(F L U X 2)$.

confidence level of 0.9 . For each object we divided the upper limit for the source counts by the local exposure time. To obtain upper limits for the luminosity, we determined a conversion factor $(C F)$ between count rate and luminosity. Because countrate and XPHOT luminosity are correlated for T Tauri stars, this was performed with a sample of 180 stars with known spectral type from our AE source catalog. The mean of the $C F$ is $3.74462 \times 10^{32} \mathrm{erg} /$ counts. The $C F$ essentially depends on absorption, and we verified that the sample of detected stars from which it was calculated has a similar mean $A_{\mathrm{J}}$ value as the sample of undetected stars to which the $C F$ was applied. The upper limit fluxes for those latter ones are listed in Table 3 together with the fluxes of the detected IC 348 members.

\section{Results}

\subsection{X-ray detection statistics}

Our combined membership list comprises 345 YSOs from spectral type B5 to M9. Only 29 of them are located outside the sky region observed with Chandra. We have X-ray detected 187 YSOs (59\% of the observed sample); see Table 4. Many of the new members identified after the study of PZ01 are too faint for detection in the existing Chandra data, even after combining the original image (Obs-ID 606) with the more recent exposures.

It is evident from the position of the 129 X-ray undetected IC 348 members in the HR diagram (Fig. 2) that these are mostly very low-mass stars and BDs. A closer look at the X-ray properties of the lowest mass objects in IC 348 is shown in Fig. 4 (left) where we display X-ray luminosities for spectral class M. Only 4 of the 39 known presumable BDs (defined here as objects with spectral type M6 and later) within the Chandra FOV are detected. The coolest X-ray detected IC 348 member has spectral type M7.5. PZ01 claimed the X-ray detection of 7 BDs and BD candidates. However, only two of them were detected by their automatic source detection routine and the other five by collecting the counts within a 3 " region around the optical position. In our new analysis we have used a more sophisticated source detection procedure. Moreover, the updates of the stellar parameters in the recent IC 348 membership studies have shifted two of the X-ray emitters previously considered BDs to earlier spectral type, i.e. stellar mass (see LSM 03). 
B. Stelzer et al.: X-ray view of IC 348 in the light of an updated cluster census
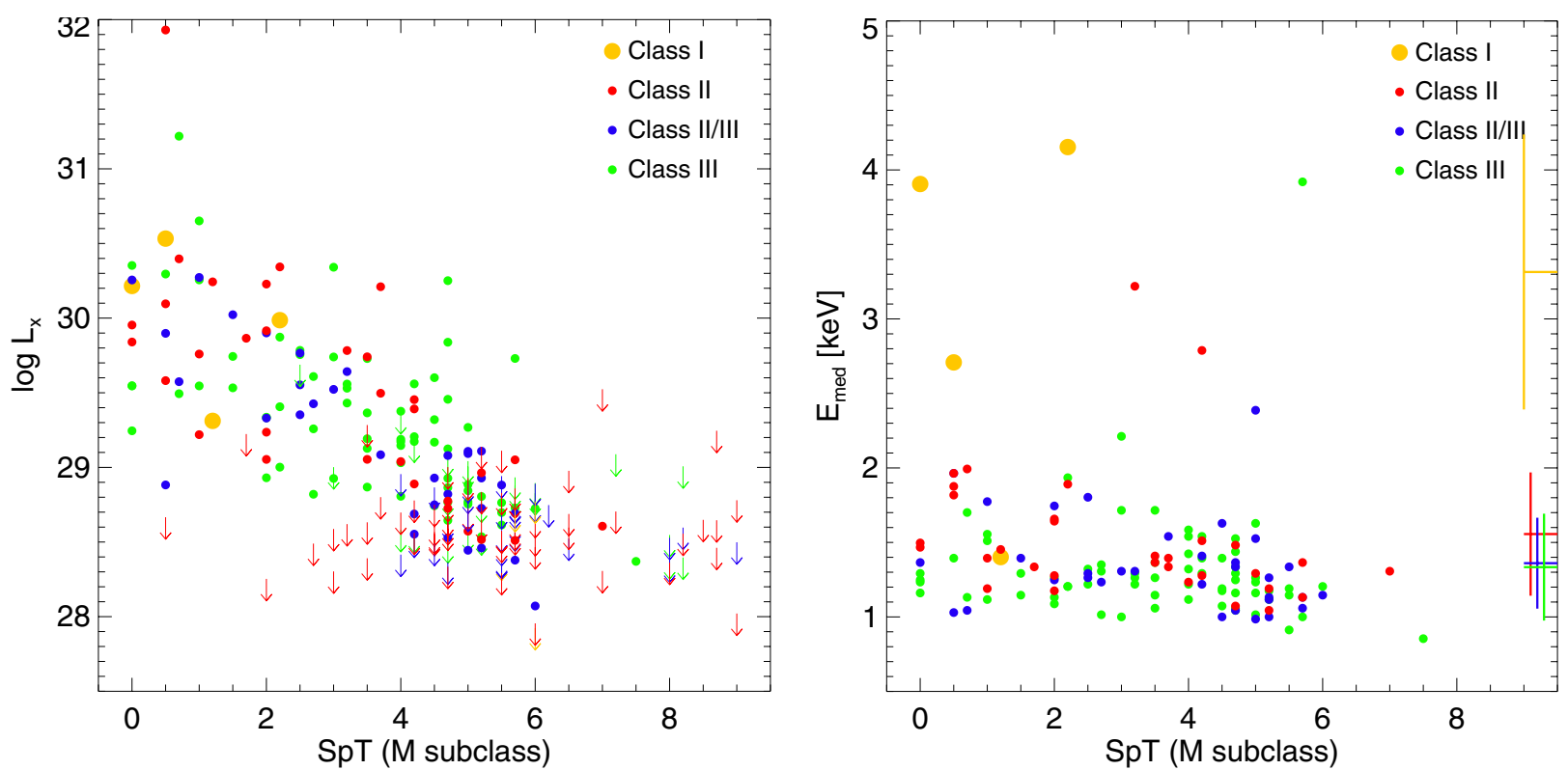

Fig. 4. X-ray luminosity and median energy for the low-mass end of IC 348 versus spectral type. The color codes refer to different YSO classes (see Fig. 1). The colored lines in the right panel represent the mean of the median energy for each YSO group irrespective of spectral type and its standard deviation, i.e. including also higher-mass stars for which individual data points are outside the plotted range.

Table 4. X-ray detection statistics for different YSO classes in IC 348.

\begin{tabular}{|c|c|c|c|c|c|c|}
\hline Mass range $\left[M_{\odot}\right]$ & & I & II & II/III & III & All \\
\hline \multicolumn{7}{|l|}{ All IC 348 members } \\
\hline & $N_{\text {det }}$ & 8 & 44 & 36 & 94 & 187 \\
\hline & $N_{\mathrm{ul}}$ & 6 & 61 & 32 & 27 & 129 \\
\hline & $\frac{N_{\text {det }}}{N}$ & 0.57 & 0.42 & 0.53 & 0.78 & 0.59 \\
\hline \multicolumn{7}{|l|}{$M<0.1$} \\
\hline & $N_{\text {det }}$ & 0 & 1 & 1 & 3 & 5 \\
\hline & $N_{\mathrm{ul}}$ & 1 & 19 & 14 & 7 & 41 \\
\hline & $\frac{N_{\mathrm{det}}}{N}$ & - & 0.05 & 0.07 & 0.30 & 0.11 \\
\hline \multicolumn{7}{|l|}{$0.1 \leq M<0.25$} \\
\hline & $N_{\text {det }}$ & 0 & 10 & 17 & 23 & 50 \\
\hline & $N_{\mathrm{ul}}$ & 2 & 28 & 15 & 13 & 58 \\
\hline & $\frac{N_{\text {det }}}{N}$ & - & 0.26 & 0.53 & 0.64 & 0.46 \\
\hline \multicolumn{7}{|l|}{$0.25 \leq M<0.6$} \\
\hline & $N_{\text {det }}$ & 0 & 11 & 9 & 30 & 51 \\
\hline & $N_{\mathrm{ul}}$ & 0 & 9 & 1 & 4 & 15 \\
\hline & $\frac{N_{\text {det }}}{N}$ & - & 0.55 & 0.90 & 0.88 & 0.77 \\
\hline \multicolumn{7}{|l|}{$0.6 \leq M<1.2$} \\
\hline & $N_{\text {det }}$ & 4 & 14 & 6 & 21 & 45 \\
\hline & $N_{\mathrm{ul}}$ & 1 & 2 & 0 & 0 & 3 \\
\hline & $\frac{N_{\mathrm{det}}}{N}$ & 0.80 & 0.88 & 1.00 & 1.00 & 0.94 \\
\hline
\end{tabular}

\section{2. $X$-rays and evolutionary stage}

Figure 5 shows color-color diagrams for Spitzer/IRAC and Spitzer/MIPS data for Lada Class I, II, II/III, and III sources. $\mathrm{X}$-ray detected and undetected stars are represented by filled and open symbols respectively. In the left panel we overplot the Class II areas defined by Megeath et al. (2004) and by Hartmann et al. (2005). As was shown already by Lada et al. (2006), the YSO classification based on the SED slope corresponds to distinct regions in the Spitzer color-color diagrams, in particular those involving [3.6]-[8.0]. This classification agrees well with the color-color selection for Class II sources introduced by Megeath et al. (2004), although the latter one includes a significant number of stars in the Class II area that are considered transition disks according to Lada et al. (2006). The Class II criteria introduced by Hartmann et al. (2005) are more conservative and include almost exclusively objects with Lada Class II flag but they miss a good fraction of them.

The X-ray detection statistics for the different YSO classes in IC 348 are summarized in Table 4 . Rows " $N_{\text {det }}$ " and " $N_{\mathrm{ul}}$ " are the number of stars detected and undetected ("upper limits") with Chandra, respectively. In the last column we give the total number of stars, regardless of YSO class. Only eight confirmed IC 348 members within the area surveyed by Chandra have no YSO classification. The detection fraction is highest for the most evolved stars, Class III, regardless of mass. For each YSO class there is a clear trend of increasing detection fraction with increasing mass. In particular, for stars with $M>0.6 M_{\odot}$ all Class II/III and III sources are detected, and the total detection statistic regardless of YSO class is $>94 \%$. On the other hand only $\sim 10 \%$ of very low-mass objects with $M<0.1 M_{\odot}$ are X-ray detected.

Luhman et al. (2003) consider membership complete in the central $16^{\prime} \times 14^{\prime}$ for $M>0.03 M_{\odot}$ and $A_{\mathrm{V}}<4 \mathrm{mag}$, but this estimate might be biased at the low-mass end against disk-less or strongly absorbed members as corroborated by the identification of more cluster members with IR spectroscopy and Spitzer mid-IR photometry, several of which in the completeness area of LSM 03 (LLM05; Muench et al. 2007). The Spitzer color selection excludes by definition the identification of Class III sources.

The X-ray luminosities of the different YSO classes are compared in Sect. 4.4. Here we point at $E_{\mathrm{med}}$ as a rough measure for spectral hardness and/or extinction. The median energy is displayed for X-ray detected M stars of IC 348 in the right panel of Fig. 4. For clarity only the spectral class M is displayed. The mean of the median energy $\left(\left\langle E_{\mathrm{med}}\right\rangle\right)$ for the full sample irrespective of spectral type is shown for each YSO class at the right end of the graph together with its standard deviation. It is striking that $\left\langle E_{\text {med }}\right\rangle$ of Class I objects is much higher than that of all other YSO classes. This is likely due to high extinction from protostellar envelopes or thick disks absorbing the soft photons. 

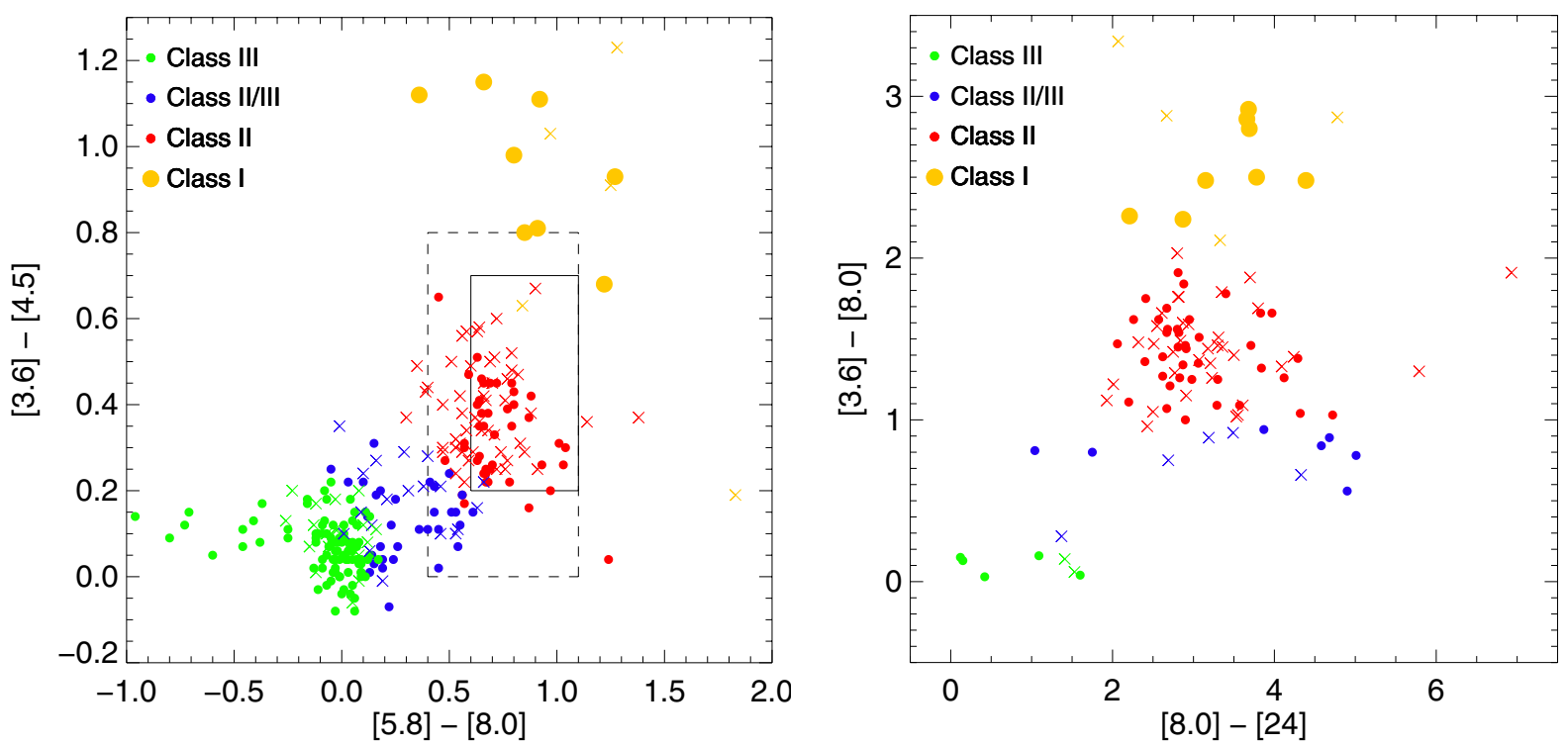

Fig. 5. Spitzer color-color diagrams for IC 348 members in the Chandra fields. X-ray detections are represented by filled circles and non-detections as $x$-points. The color codes for different SED YSO classes are the same as in the previous figures and, for convenience, defined again in the top left of each panel. The boxes in the left figure show the areas defining Class II according to Megeath et al. (2004) and Hartmann et al. (2005); dashed and solid lines, respectively.

\subsection{X-ray and bolometric luminosity}

The ratio between $\mathrm{X}$-ray and bolometric luminosity is thought to be a crucial indicator for the level of magnetic activity in young stars. From X-ray surveys in various star-forming regions it has emerged that the characteristic emission level of most low-mass pre-main sequence stars is concentrated between $\log \left(L_{\mathrm{x}} / L_{\mathrm{bol}}\right) \approx-3$ and -4 but with a spread of at least 3 dex, and there are indications for a slight decline of the $\log \left(L_{\mathrm{x}} / L_{\mathrm{bol}}\right)$ level with decreasing mass (e.g. Flaccomio et al. 2003; Preibisch et al. 2005a,b; Grosso et al. 2007).

\subsubsection{Sample without distinction of YSO class}

Figure 6 shows the $L_{\mathrm{x}}$ vs. $L_{\text {bol }}$ diagram for IC 348. The sample of known IC 348 members extends to lower bolometric luminosities and masses than that of Orion, possibly caused by both environmental and observational effects (e.g. higher extinction in Orion) and the closer distance. We carried out a linear regression fit for the range $\log \left(L / L_{\odot}\right)=-1.5 \ldots+0.5$ using the EM algorithm in ASURV (Feigelson \& Nelson 1985). Our choice for the luminosity cutoffs are motivated by the properties of the IC 348 sample (avoiding at the high end X-ray faint massive stars outside the mass range covered by the Baraffe et al. (1998) models and avoiding to be dominated by upper limits in X-rays at the low end). The result is overplotted in Fig. 6 together with the best fit obtained by Preibisch et al. (2005a) for the ONC in a similar range of bolometric luminosities. The ONC study was performed for stars with $\log \left(L / L_{\odot}\right)<1$ and without lower cutoff to the bolometric luminosity. However, effectively the ONC sample extends down to $\log \left(L / L_{\odot}\right)=-1.5$, i.e. the same as our lowluminosity cutoff. With respect to Preibisch et al. (2005a), we have chosen a lower value for the luminosity cutoff at the high end because at the age of IC 348 stars with $\log \left(L / L_{\odot}\right)>0.5$ are mostly radiative (cf. Fig. 2) and, therefore, their X-ray emission - if any - may not have the same origin as in lower-mass stars.

The slope we determined for IC 348 in the luminosity range described above $(1.27 \pm 0.08)$ is significantly steeper than the one measured from COUP for the ONC $(1.04 \pm 0.06)$ and from

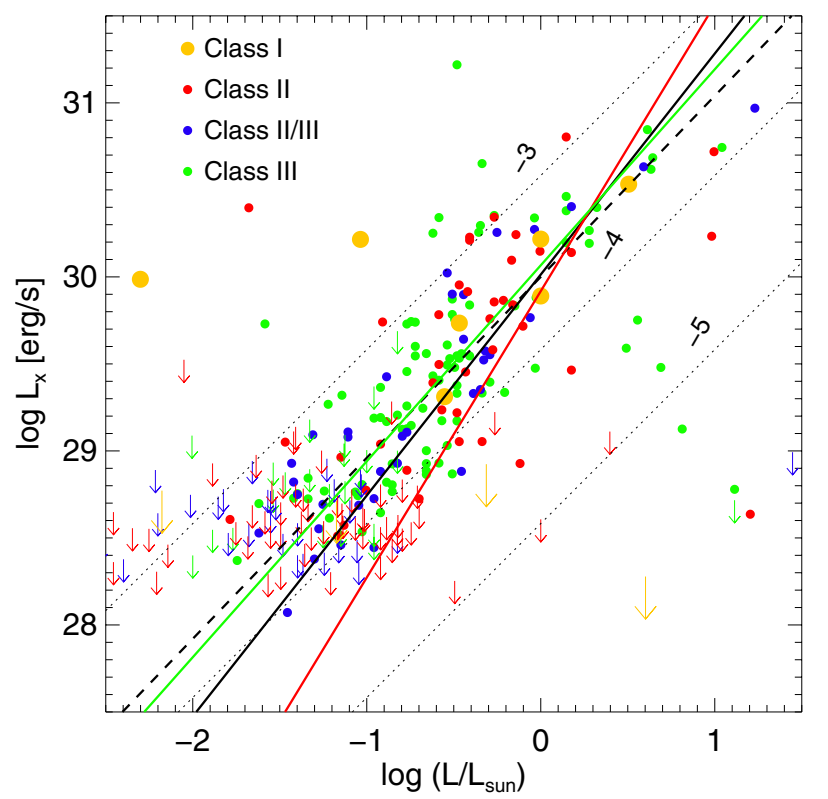

Fig. 6. X-ray versus bolometric luminosity (a small number of objects, not relevant for this study, falls outside the plotted $L_{\text {bol }}$ range); dotted lines represent constant $L_{\mathrm{x}} / L_{\mathrm{bol}}$ ratios $10^{-3}, 10^{-4}$ and $10^{-5}$; the dashed line is the linear regression fit derived by Preibisch et al. (2005a) for the ONC corresponding to $\log \left(L_{\mathrm{x}} / L_{\mathrm{bol}}\right)=-3.7$; the solid lines are linear regression fits to the IC 348 members in the luminosity range $\log \left(L / L_{\odot}\right)=-1.5 \ldots+0.5$, which corresponds roughly to the upper mass boundary of the Baraffe et al. (1998) models: black, red and green are for the whole sample, only Class II and only Class III sources, respectively.

XEST for Taurus $(1.05 \pm 0.06$; Telleschi et al. 2007). While in Orion and Taurus the linear fit of $L_{\mathrm{x}}$ vs. $L_{\mathrm{bol}}$ is consistent with a constant $L_{\mathrm{x}} / L_{\mathrm{bol}}$ ratio, in IC 348 we observe a decrease of $L_{\mathrm{x}} / L_{\mathrm{bol}}$ with decreasing bolometric luminosity. A possible reason may be the particular distribution of YSO types and their different X-ray luminosities. Note, however, that the COUP and XEST samples we refer to are not directly equivalent to our 
B. Stelzer et al.: X-ray view of IC 348 in the light of an updated cluster census
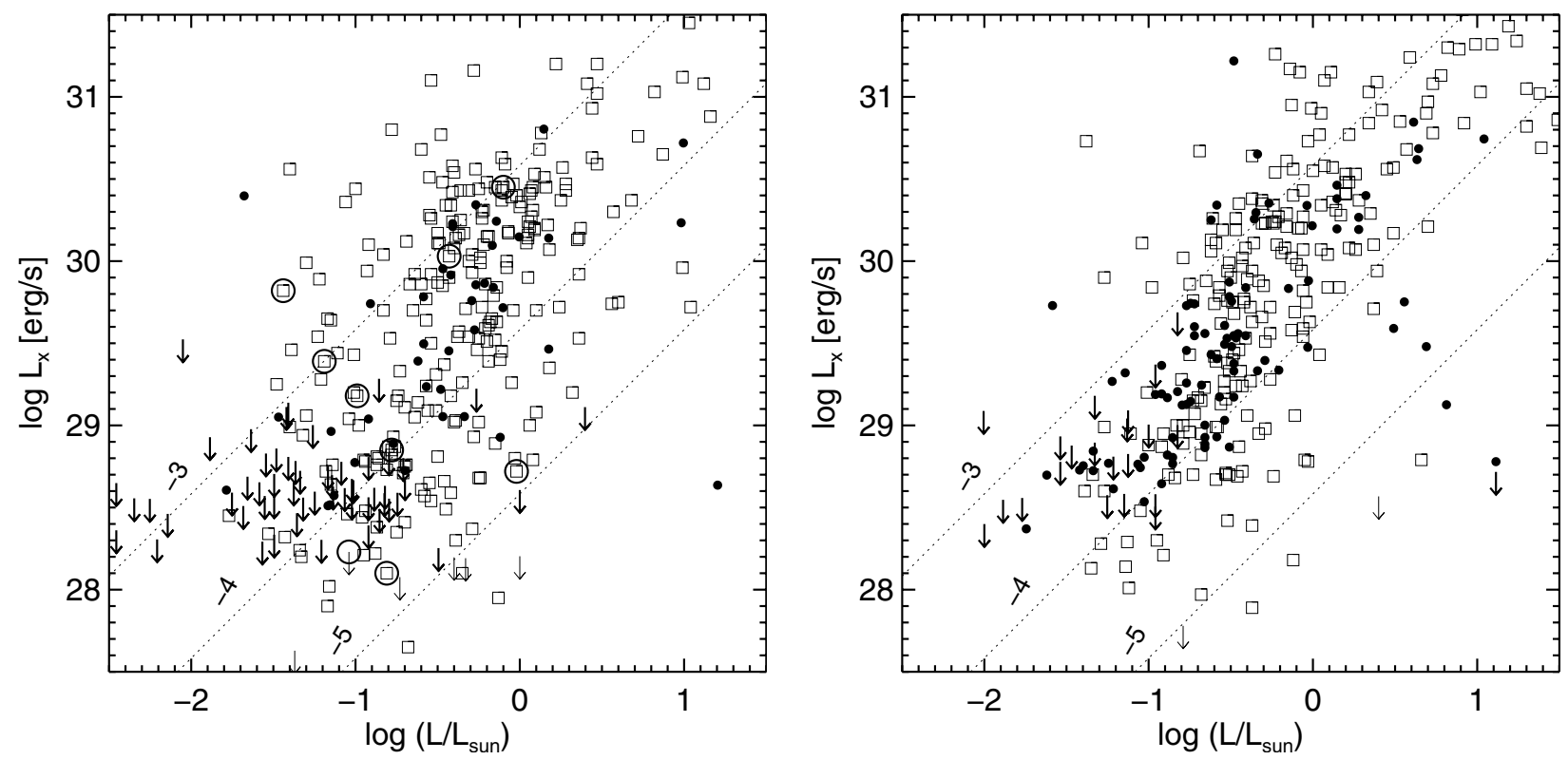

Fig. 7. X-ray versus bolometric luminosity for IC 348 (filled, thick plotting symbols) compared to the ONC (open, thin plotting symbols): (left) Class II, (right) - Class III. Stars with indications for a disk warp provoking temporarily high extinction (AA Tau-like stars) are highlighted with large circles.

samples in IC 348 because of different ways the subsamples were defined. Preibisch et al. (2005a) distinguish accretors from nonaccretors on the basis of $8542 \AA$ calcium emission. Similarly, Telleschi et al. (2007) separate accretors from non-accretors on the basis of $\mathrm{H} \alpha$ emission, while we have chosen to work with the Spitzer YSO classification because it yields the largest sample size.

\subsubsection{Class II vs. Class III and comparison with the ONC}

When the same analysis is carried out separately for the subsamples of Class II and Class III sources in IC 348 we find slopes of $1.65 \pm 0.22$ and $1.13 \pm 0.11$, respectively. These results are graphically demonstrated in Fig. 6. To summarize, we find that

- the $L_{\mathrm{x}}-L_{\mathrm{bol}}$ relation of Class III objects in IC 348 agrees with that of the non-accretors in the $\mathrm{ONC}$ and is roughly constant across the examined range of bolometric luminosities;

- the Class II sources in IC 348 show a steeper slope, i.e. decreasing $L_{\mathrm{x}}-L_{\mathrm{bol}}$ ratio toward fainter stars. IC 348 hosts a large number of Class II sources with low luminosities (i.e. low masses) - see e.g. Table 4 - and these objects have low $\mathrm{X}$-ray luminosities or they are not detected.

Only recently YSO classifications for ONC stars based on Spitzer/IRAC photometry have become available. We extracted these data from the YSOVAR database 4 . In Fig. 7 we compare the X-ray vs. bolometric luminosities for IC 348 with those of the ONC separately for Class II and Class III sources.

According to Fig. 7(left), in contrast to IC 348 there is no evidence for an X-ray deficiency of low-luminosity diskbearing stars in the ONC. However, a look at the HR diagram for the ONC, presented in Fig. 8, suggests that the bolometric luminosities of these stars (i.e. the ones with $\log L_{\mathrm{bol}} \sim-1 \ldots-1.5$ ) may be underestimated. This group is characterized by an obvious excess of Class II sources with isochronal age around $10 \mathrm{Myr}$ or more in the HR diagram, in contradiction with the general paradigm that Class II stars are on average less evolved than

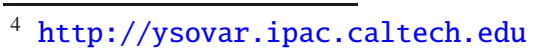

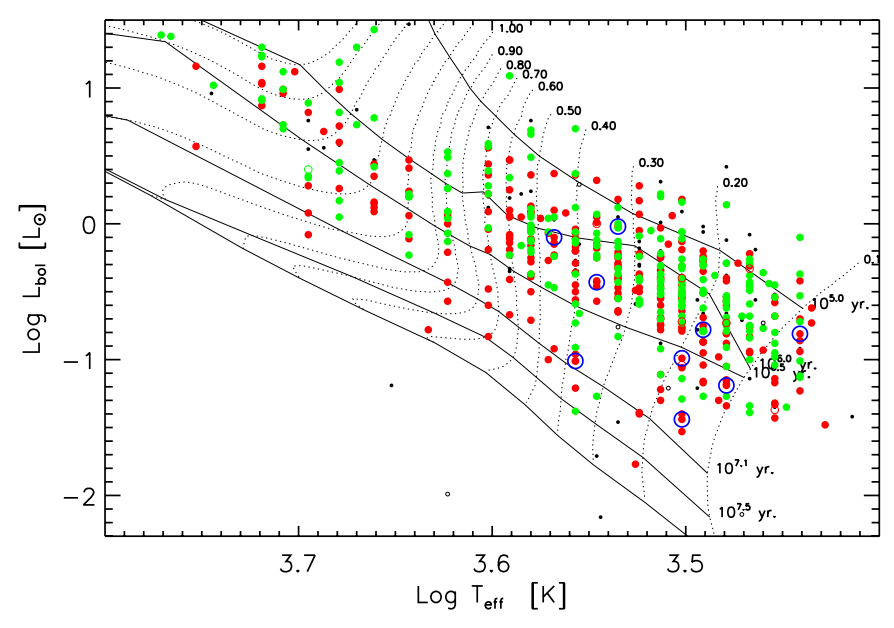

Fig. 8. HR diagram for the optical COUP sample on the Siess et al. (2000) models. Class III (green; light gray in b/w print) and Class II (red; dark gray in b/w print), AA Tau like stars (large blue annuli) and IC 348 members without YSO classification (small black points).

Class III stars as well as with plausible timescales for disk dissipation of just a few Myr. We hypothesize that many of these low-luminosity, low-mass Class II objects are either observed in scattered light, the central star being fully obscured by an edgeon disk, or partially obscured by disk warps or other circumstellar structures the so-called AA Tau phenomenon. A number of AA Tau-like stars in the ONC were identified by MoralesCalderón et al. (2011) and are marked in Fig. 8 by large annuli. Not all of the cluster members with untypically low bolometric luminosity for their effective temperature display the AA Tau features but this does not contradict our hypothesis because occultations by disk warps are transient phenomena. Indeed, these objects were identified by Morales-Calderón et al. (2011) on the basis of their time-variability. Moreover, some of these objects may be permanently obscured systems with an edge-on disk. Both of the described scenarios would imply that the extinction correction for these stars has been significantly underestimated, 
thus placing the true position of the stars in the $L_{\mathrm{x}}-L_{\mathrm{bol}}$ diagram farther to the right, which agrees better with the trend that we see in IC 348. That none of the Class II stars with low $L_{\text {bol }}$ but high $L_{\mathrm{x}}$ are present in IC 348 might be explained by the older age with respect to the ONC, going along with the dispersal of the circumstellar material that provides the extinction.

We now consider the Class III sources (Fig. 7 right). In contrast to the analysis of Preibisch et al. (2005a) for non-accretors, the ONC Class III sample shows evidence for a steeper $L_{\mathrm{x}}-L_{\mathrm{bol}}$ relation than the one corresponding to a constant luminosity ratio. This is in line with the result for Class II sources considering the hypothesis made above concerning AA Tau-like stars. We were unable to detect this deficiency of low-luminosity X-ray faint Class III sources in IC 348 but this may be because of the limited sample size. From Fig. 7 it appears that the IC 348 and ONC Class III distributions are quite similar.

\subsection{X-ray luminosity functions}

Cumulative distributions of the X-ray luminosities are traditionally used to compare subsamples of pre-main sequence stars. In the past, the X-ray luminosity functions (XLF) derived for various star-forming regions have been vigourously debated, because it could not be established if circumstellar matter influences these distributions. Most studies that distinguished accreting and non-accreting stars on basis of the strength of their $\mathrm{H} \alpha$ emission found that cTTS have systematically lower X-ray luminosities than wTTS (Stelzer \& Neuhäuser 2001; Flaccomio et al. 2003). On the other hand, no differences were found between stars with and without disks as diagnosed by IR excess (e.g. PZ01).

We have constructed cumulative distributions of both the X-ray luminosity (i.e. the XLFs) and the $L_{\mathrm{x}} / L_{\text {bol }}$ ratio. We computed XLFs for YSOs of different SED classes (to examine the influence of disks) and for different strength of $\mathrm{H} \alpha$ emission (to examine the influence of accretion). To avoid biases related to the known dependence of $L_{\mathrm{x}}$ on stellar mass, the analysis was carried out in three mass bins chosen such that they sample adequately the low-mass IC 348 population: $0.1-0.25 M_{\odot}$, $0.25-0.6 M_{\odot}, 0.6-1.2 M_{\odot}$. The significance of the results is verified with two-sample tests that yield the probability for rejecting the hypothesis that the observed luminosity distributions are drawn from the same parent distributions. Both the cumulative distributions and the two-sample tests were computed within the ASURV environment. The calculations take into account the upper limits for undetected stars.

The results are displayed in Figs. 9 and 10. In the left-hand panels we compare the XLFs of Class II, II/III, and III sources. Class I objects are not considered because of poor statistics. There is a pronounced difference in the X-ray luminosities of the three YSO classes for the lowest mass bin $\left(0.1-0.25 M_{\odot}\right)$, where Class III sources have higher $L_{\mathrm{x}}$ than both Class II/III and Class II and the latter two classes are indistinguishable. This is also the mass range with the highest number statistics comprising 106 stars. However, these low-mass samples have a large number of upper limits. A marginal difference between Class II objects and the other two YSO groups is found for the intermediate mass bin $\left(0.25-0.6 M_{\odot}\right)$ only in the logrank test while all other types of two-sample tests could not detect a difference with better than $90 \%$ probability. For the highest mass bin $\left(0.6-1.2 M_{\odot}\right)$ the XLFs of the three types of YSOs cannot be distinguished. The graphical results are supported by the twosample tests. The statistics are summarized in Table 5 where " $N$ " is the total number of stars and " $N_{\mathrm{ul}}$ " is the number of undetected stars. The luminosities refer to the median of each sample, and the numbers in the last three columns represent probabilities for two distributions being different obtained from two-sample tests.

There is a marked difference also in terms of $L_{\mathrm{x}} / L_{\mathrm{bol}}$ between the three YSO classes only in the lowest mass bin. In contrast to the XLFs, where Class II/III objects behave similar to the Class II sources, in $L_{\mathrm{x}} / L_{\mathrm{bol}}$ the Class II/III objects are more similar to the Class III sources (compare uppermost left panels in Figs. 9 and 10). Again only the logrank test gives Class II objects different from Class III for the intermediate mass bin. All other distributions of $L_{\mathrm{x}} / L_{\mathrm{bol}}$ for higher masses are indistinguishable. These results confirm our findings from Fig. 6 that the $L_{\mathrm{x}}-L_{\mathrm{bol}}$ relation in IC 348 is steeper for Class II than for Class III sources.

An analogous investigation was performed for cTTS and wTTS, distinguished by the equivalent width of $\mathrm{H} \alpha$ as described in Sect. 2.2. The XLFs and the cumulative distributions for $L_{\mathrm{x}} / L_{\mathrm{bol}}$ for cTTS and wTTS are shown in the right panels of Figs. 9 and 10 for the same mass bins that we examined for the samples classified by their IR excess. Again, a significant difference between the groups is found for the lowest mass bin with wTTS being more X-ray luminous than cTTS; see Table 6 for a summary of the results. In the intermediate mass bin, where the Class II stars were different from the other two samples only in the logrank test, the cTTS and the wTTS are different at high significance for all two-sample tests if $L_{\mathrm{x}}$ is considered but, again, only in the logrank test if $L_{\mathrm{x}} / L_{\mathrm{bol}}$ is considered.

Note that the IR excess and the $\mathrm{H} \alpha$ emission samples are not identical and their comparison is not straightforward. The number of stars with measured $\alpha_{3-8 \mu \mathrm{m}}$ is larger than those with $\mathrm{H} \alpha$ data. For the lowest mass bin, most of the Class II/III sources belong to the wTTS group. However, there are also several Class II sources that are classified as non-accretors. We have shown that the trend of a mass dependence of the X-ray emission level throughout the various evolutionary stages of YSOs is robust against details of the sample definition.

\subsection{X-ray luminosity and mass accretion}

In the past, differences between the X-ray luminosities of cTTS and wTTS have been ascribed to a possible connection between $\mathrm{X}$-ray emission and accretion. The interpretations have involved opposite views: In one scenario X-ray emission is suppressed by accretion, e.g. by a direct influence of accretion onto the dynamo efficiency through reduced convection or through reduced coronal heating as a result of the higher plasma densities involved in the accretion columns (e.g. Preibisch et al. 2005a) or by occulting the X-ray emitting corona (Gregory et al. 2007). In the alternative picture X-ray emission determines whether accretion takes place or not through photoevaporation of disks. This latter hypothesis has been bolstered by an empirical anticorrelation of X-ray luminosity and mass accretion rate observed in COUP (Drake et al. 2009).

Only 18 IC 348 members have a measured mass accretion rate. In Fig. 11 we present the relation between $L_{\mathrm{x}}$ and $\dot{M}_{\text {acc }}$ for IC 348. Note that at least two stars in IC 348 with "anemic" disks are accreting. The top panel of this figure shows that the apparent positive correlation between $L_{\mathrm{x}}$ and $\dot{M}_{\text {acc }}$ is a result of the well-known dependence of mass accretion rate on stellar mass (see e.g. Natta et al. 2006). Three of the objects in the group of strong accretors (empirical dividing line of $\log \dot{M}_{\text {acc }}=-9$ ) have no mass assigned because they are outside the mass range covered by the Baraffe et al. (1998) models $\left(M>1.2 M_{\odot}\right)$. All in all, however, all stars in this group have similar mass but span a range of two decades in accretion rates and more than one decade 
B. Stelzer et al.: X-ray view of IC 348 in the light of an updated cluster census


Fig. 9. Cumulative distributions of X-ray luminosity for different subgroups of YSO in three mass bins (from top to bottom). The left panels represent three YSO classes based on IR excess (red dotted - Class II, blue dashed - Class II/III, and green solid - Class III) and the right panels represent YSO classes based on H $\alpha$ emission (green solid - wTTS and red dotted - cTTS).

in X-ray luminosity. The Spearmans test yields no evidence for a correlation between the two parameters for the IC 348 members with strong accretion. However, the small sample size precludes any firm conclusions.

\section{Discussion and conclusions}

Our combined analysis of all existing Chandra observations provides the so far deepest X-ray survey for IC 348. It is difficult to give a number for the sensitivity limit due to the partial overlap of the available images. The exposure time for the deepest part of the merged mosaic is $180 \mathrm{ks}$ and the faintest X-ray detected cluster member has a luminosity of $\log L_{\mathrm{X}}[\mathrm{erg} / \mathrm{s}]=28.1$. Our detection rate for BDs in IC 348 is $10 \%$, in an area surveyed by Chandra that misses only 2 out of 41 substellar objects.

The magnetic activity at and beyond the low-mass end of the main-sequence is generally poorly constrained owing to a lack of sufficiently sensitive observations in the X-ray regime. Even COUP, the deepest current X-ray image of a star-formation region, has yielded detections for only $26 \%$ of the known BDs, presumably because of the considerable extinction in the ONC. We caution that the COUP BD sample may be contaminated by a few objects with mass above the substellar limit because the BD classification was based mostly on near-IR spectra that are known to yield systematically later spectral types compared to optical spectra (see Preibisch et al. 2005b, for more details on the sample definition). Nine BDs were detected in Taurus during 

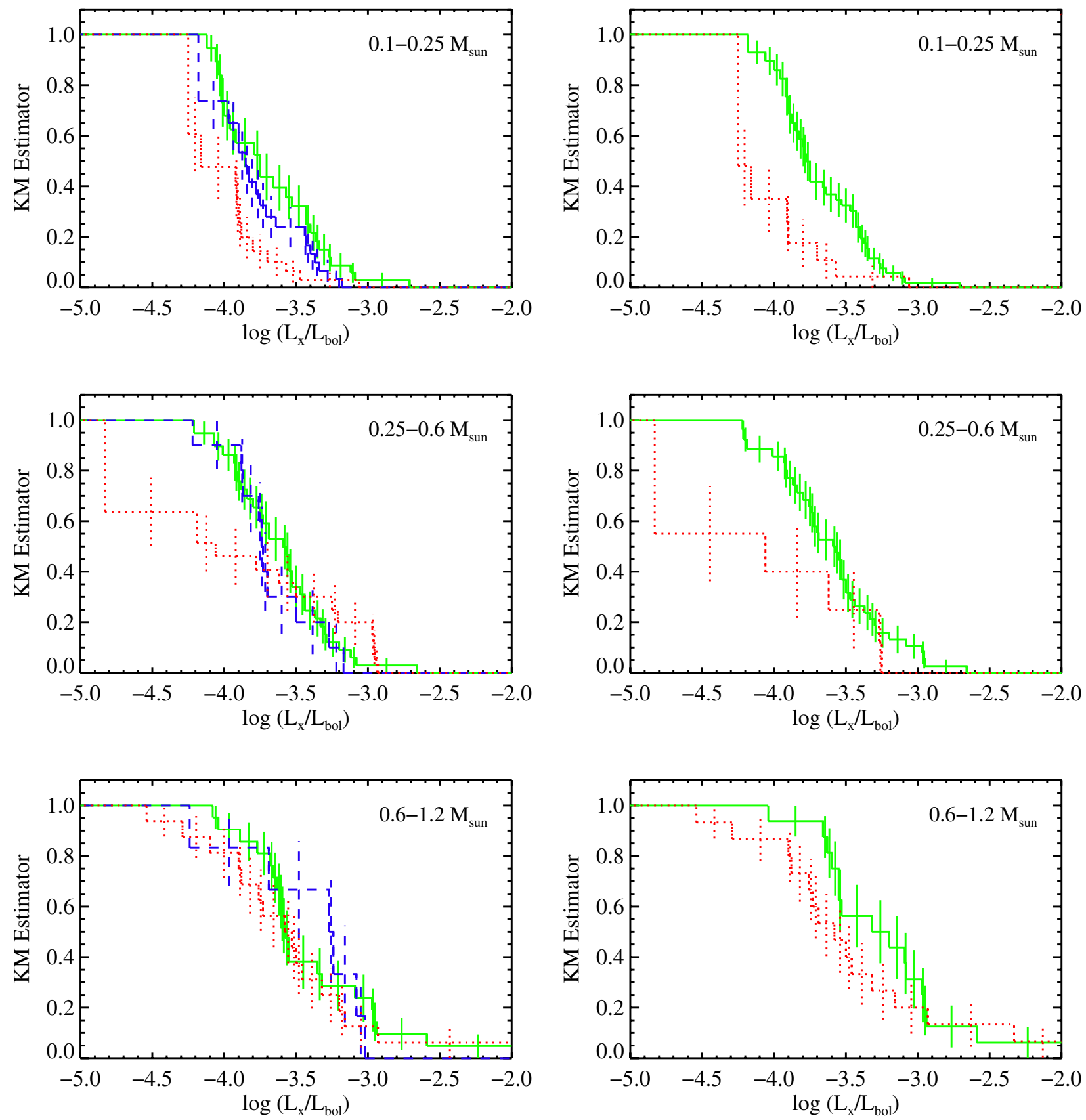

Fig. 10. Same as Fig. 9 but for the fractional X-ray luminosity $\log \left(L_{\mathrm{x}} / L_{\mathrm{bol}}\right)$.

Table 5. Statistics for detections, median of the logarithm of X-ray luminosity and $L_{\mathrm{x}} / L_{\mathrm{bol}}$ ratio, and 2-sample tests of different YSO classes.

\begin{tabular}{|c|c|c|c|c|c|c|c|c|c|}
\hline \multirow[b]{2}{*}{$M\left[\mathrm{M}_{\odot}\right]$} & \multicolumn{2}{|c|}{ Class III } & \multicolumn{2}{|c|}{ Class II/III } & \multicolumn{2}{|c|}{ Class II } & \multirow{2}{*}{$\begin{array}{c}\text { 2S-Test } \\
\text { Class III-II } \\
\text { Prob.[\%] }\end{array}$} & \multirow{2}{*}{$\begin{array}{c}2 \mathrm{~S}-\mathrm{Test} \\
\text { Class III-II/III } \\
\text { Prob.[\%] }\end{array}$} & \multirow{2}{*}{$\begin{array}{c}\text { 2S-Test } \\
\text { Class II/III-II } \\
\text { Prob.[\%] }\end{array}$} \\
\hline & $N / N_{\mathrm{ul}}$ & $\log L_{\mathrm{x}}$ & $N / N_{\mathrm{ul}}$ & $\log L_{\mathrm{x}}$ & $N / N_{\mathrm{ul}}$ & $\log L_{\mathrm{X}}$ & & & \\
\hline $0.1-0.25$ & $36 / 13$ & 28.76 & $32 / 15$ & 28.51 & $38 / 28$ & 28.47 & $>99$ & $>97$ & $<90$ \\
\hline $0.25-0.6$ & $34 / 4$ & 29.20 & $10 / 1$ & 29.43 & $20 / 9$ & 29.04 & $<90^{a}$ & $<90$ & $<90^{a}$ \\
\hline $0.6-1.2$ & $21 / 0$ & 29.55 & $6 / 0$ & 29.90 & $16 / 0$ & 29.76 & $<90$ & $<90$ & $<90$ \\
\hline$M\left[M_{\odot}\right]$ & $N / N_{\mathrm{ul}}$ & $\log L_{\mathrm{x}} / L_{\text {bol }}$ & $N / N_{\mathrm{ul}}$ & $\log L_{\mathrm{x}} / L_{\mathrm{bol}}$ & $N / N_{\mathrm{ul}}$ & $\log L_{\mathrm{x}} / L_{\mathrm{bol}}$ & Prob.[\%] & Prob.[\%] & Prob.[\%] \\
\hline $0.1-0.25$ & $36 / 13$ & -3.78 & $32 / 15$ & -3.87 & $38 / 28$ & -4.18 & $>99$ & $<90$ & $>97$ \\
\hline $0.25-0.6$ & $34 / 4$ & -3.60 & $10 / 1$ & -3.74 & $20 / 9$ & -4.15 & $<90^{b}$ & $<90$ & $<90$ \\
\hline $0.6-1.2$ & $21 / 0$ & -3.59 & $6 / 0$ & -3.27 & $16 / 0$ & -3.58 & $<90$ & $<90$ & $<90$ \\
\hline
\end{tabular}

Notes. ${ }^{(a)}$ The logrank test gives probabilities of $>97 \%$ and $>93 \%$ for Class III vs. Class II and Class II/III vs. Class II. ${ }^{(b)}$ The logrank test gives a probability of $>99 \%$ for Class III vs. Class II. 
Table 6. Statistics for detections, median of the logarithm of X-ray luminosity and $L_{\mathrm{x}} / L_{\mathrm{bol}}$ ratio, and two-sample tests for cTTS and wTTS.

\begin{tabular}{lccccc}
\hline \hline & \multicolumn{2}{c}{ wTTS } & \multicolumn{2}{c}{ cTTS } & 2S-Test \\
& wTTS - cTTS \\
$M\left[M_{\odot}\right]$ & $N / N_{\mathrm{ul}}$ & $\log L_{\mathrm{x}}$ & $N / N_{\mathrm{ul}}$ & $\log L_{\mathrm{x}}$ & Prob.[\%] \\
\hline $0.1-0.25$ & $55 / 18$ & 28.74 & $26 / 20$ & 28.18 & $>99$ \\
$0.25-0.6$ & $38 / 3$ & 29.38 & $8 / 4$ & 28.51 & $>95$ \\
$0.6-1.2$ & $16 / 0$ & 29.76 & $15 / 0$ & 29.78 & $<90$ \\
\hline$M\left[M_{\odot}\right]$ & $N / N_{\mathrm{ul}}$ & $\log \frac{L_{\mathrm{x}}}{L_{\mathrm{bol}}}$ & $N / N_{\mathrm{ul}}$ & $\log \frac{L_{\mathrm{x}}}{L_{\mathrm{bol}}}$ & Prob.[\%] \\
\hline $0.1-0.25$ & $55 / 18$ & -3.78 & $26 / 20$ & -4.09 & $>99$ \\
$0.25-0.6$ & $38 / 3$ & -3.59 & $8 / 4$ & -4.57 & $<90^{a}$ \\
$0.6-1.2$ & $16 / 0$ & -3.32 & $15 / 0$ & -3.64 & $<90$ \\
\hline
\end{tabular}

Notes. ${ }^{(*)}$ The logrank test gives a probability of $>96 \%$.

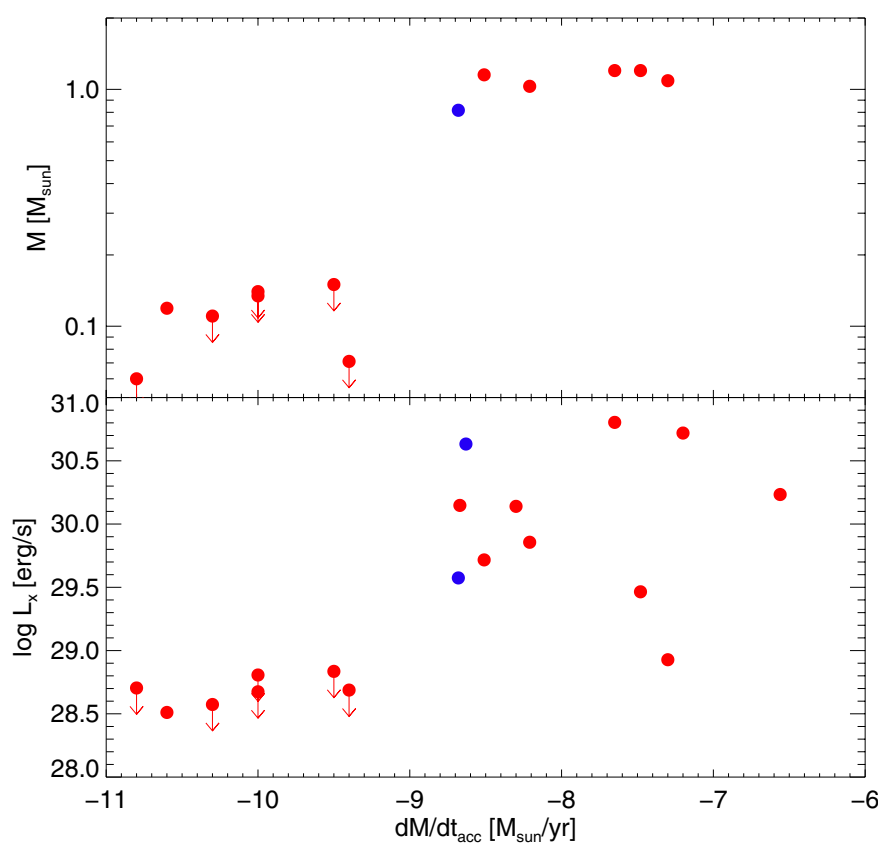

Fig. 11. X-ray luminosity and mass versus mass accretion rate. Colors define YSO classes as in Fig. 1.

the XEST (Grosso et al. 2007) using the same definition for BDs as we do in this paper for IC 348 (spectral type M6 and later). The XEST sample comprised 17 BDs known at the time, i.e. the X-ray detection rate was about $50 \%$. It is important to note that many of the X-ray detected BDs in Taurus have untypically high bolometric luminosities that place them above the $1 \mathrm{Myr}$ isochrone of the Baraffe et al. (1998) models (see e.g. Fig. 2 in Grosso et al. 2007). As a consequence, for a given $L_{\mathrm{x}} / L_{\mathrm{bol}}$ level their X-ray luminosity is higher than for IC 348 BDs which explains the higher detection rate.

We have examined cumulative distributions of absolute and fractional X-ray luminosity in three mass ranges: $0.1-0.25$, $0.25-0.6$, and $0.6-1.2 M_{\odot}$. The choice of these mass bins was driven by the requirement to have sufficient stars in each subsample for a statistical analysis. The well-known increase of the median X-ray luminosity with increasing mass underlines once more that the distinction of different mass bins is mandatory. However, the details of the derived XLFs also depend on the choice of the boundaries for the mass bins, and more generally on the pre-main sequence models. For this reason, results for different star-forming regions are difficult to compare. In COUP, e.g., the Siess et al. (2000) models were used, and the mass bins that were chosen are different from ours motivated by the high number of stars with solar mass and above.

In addition to the question with stellar masses, the literature provides differing definitions for characterizing the evolutionary stage of pre-main sequence stars. In COUP, e.g., disks were characterized by near-IR excess effectively biased toward warmer disks with respect to our Spitzer YSO classification. Other COUP subsamples represent accreting and non-accreting stars separated by the strength of calcium emission. In XEST YSOs were separated into accretors and non-accretors on the basis of $\mathrm{H} \alpha$ emission, while no disk-status was assigned to the stars. We have examined for IC 348 both the influence of the presence of disks and of ongoing accretion on the X-ray emission level. Our results hold for both types of classifications. In particular, we find that differences exist between groups of YSOs in different evolutionary stages at low masses, while they disappear at higher mass. This confirms a trend that was seen in COUP (Preibisch et al. 2005a). An opposite tendency was claimed in XEST: different XLFs for cTTS and wTTS at $M>0.3 M_{\odot}$ but not for lower masses (Telleschi et al. 2007). This result does not hold for IC 348 according to our data.

The trends that we see in the XLFs of IC 348 directly reflect the deficiency of X-ray luminosity in very low-mass diskbearing stars responsible for the drop of the $L_{\mathrm{X}} / L_{\mathrm{bol}}$ ratio toward fainter, less massive stars. We have compared this result to ONC samples that were constructed by combining the recent YSO classification with COUP-derived parameters, and find a decline of the $L_{\mathrm{x}} / L_{\mathrm{bol}}$ level toward low-luminosity for the diskless stars in the ONC. In disk-bearing stars of the ONC the scatter is large, possibly owing to biases related to the difficulty of correcting the observed luminosities for high circumstellar extinction that is ubiquitous at the young age of Orion.

Some scenarios for a decrease of $L_{\mathrm{x}} / L_{\mathrm{bol}}$ level toward lower masses are: (i) transition from a solar-like to a convective dynamo, combined with a lower efficiency of the latter that results in decreased X-ray production; (ii) smaller co-rotation radius for lower-mass stars and the ensuing centrifugal disruption of the corona (Jardine et al. 2006); (iii) a mass-dependent fraction of stars with X-ray emission suppressed by accretion (Flaccomio et al. 2003). Concerning (ii), we note that indeed the IC 348 stars in our lowest mass bin all have short rotation periods $(\leq 5 \mathrm{~d})$ while the period distribution is much broader for higher mass stars, which is discussed in Cieza \& Baliber (2006). Alexander et al. (in prep.) present a detailed study of the rotation/activity connection in IC 348 and find no significant differences between stars in the saturated and in the super-saturated regime. Moreover, rotational effects should affect both disk-bearing and diskless stars in the same manner. Scenario (iii) is the only one that distinguishes between YSOs of given mass but in different evolutionary phases and would explain the different XLFs of very low-mass Class II and Class III objects. The search for a direct connection between mass accretion rate and X-ray luminosity could shed light on this question but, at present, it is hampered because of the small number of IC 348 members with accretion measures. In the available sample of 12 objects we find no relation between $\dot{M}_{\text {acc }}$ and $L_{\mathrm{x}}$, except for the trend that can be ascribed to the well-known dependence of both parameters on mass.

To summarize, we have examined one of the deepest available X-ray data sets on a young stellar cluster. Thanks to its rich population of very low-mass cluster members IC 348 provides an excellent opportunity to study the X-ray activity level at the boundary and beyond the substellar limit. We have established for the first time with statistical significance the non-constant 


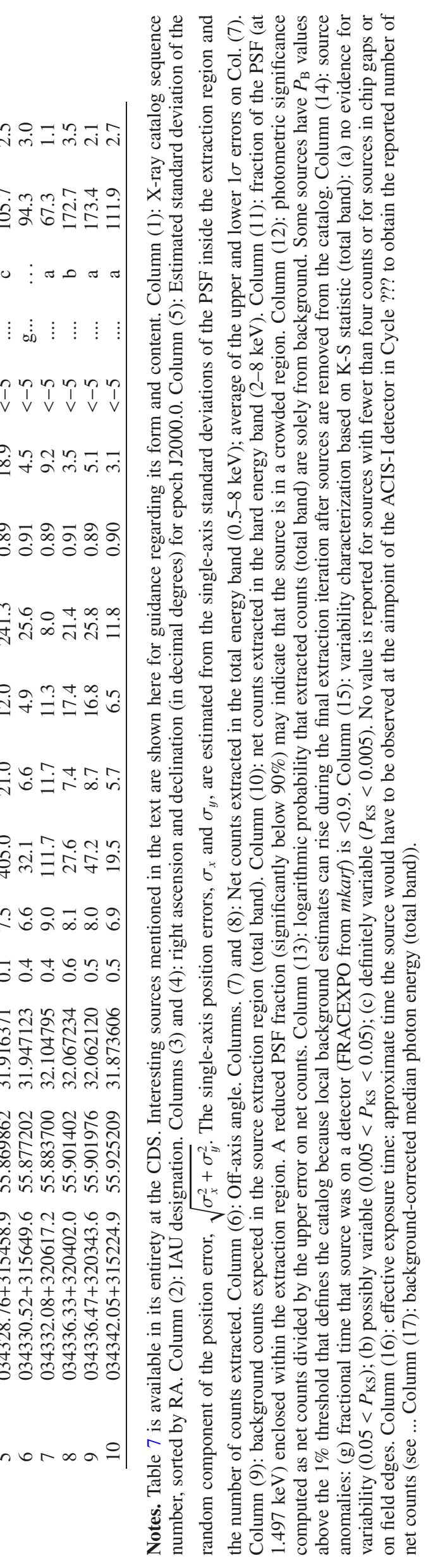


behavior of $L_{\mathrm{x}} / L_{\mathrm{bol}}$ for the very low-mass pre-main sequence. Although the sensitivity of the X-ray data is comparable to that of other recent studies of star-forming regions such as XEST and COUP, the well-characterized cluster census way down into the $\mathrm{BD}$ regime results in a low $\mathrm{X}$-ray detection rate for very lowmass objects and the low-luminosity end of the X-ray data of IC 348 continues to be dominated by insufficiently constrained upper limits. A much longer exposure would substantially increase the X-ray census at the low-mass end of the stellar sequence in IC 348. Additional studies of accretion rates in IC 348 would help to understand the interplay between accretion and $\mathrm{X}$-ray emission.

Acknowledgements. We would like to thank the referee, M. Güdel. B.S., G.M., E.F. and S.S. acknowledge financial contribution from the agreement ASI-INAF I/009/10/0. This work was also supported by the Munich Cluster of Excellence "Origin and Structure of the Universe".

\section{References}

Baraffe, I., Chabrier, G., Allard, F., \& Hauschildt, P. H. 1998, A\&A, 337, 403 Broos, P. S., Feigelson, E. D., Townsley, L. K., et al. 2007, ApJS, 169, 353 Broos, P. S., Townsley, L. K., Feigelson, E. D., et al. 2010, ApJ, 714, 1582 Burgess, A. S. M., Moraux, E., Bouvier, J., et al. 2009, A\&A, 508, 823 Chabrier, G., Baraffe, I., Allard, F., \& Hauschildt, P. 2000, ApJ, 542, 464 Cieza, L., \& Baliber, N. 2006, ApJ, 649, 862

Currie, T., \& Kenyon, S. J. 2009, AJ, 138, 703

Dahm, S. E. 2008, AJ, 136, 521

de Geus, E. J., de Zeeuw, P. T., \& Lub, J. 1989, A\&A, 216, 44

Drake, J. J., Ercolano, B., Flaccomio, E., \& Micela, G. 2009, ApJ, 699, L35

Eislöffel, J., Froebrich, D., Stanke, T., \& McCaughrean, M. J. 2003, ApJ, 595, 259

Feigelson, E. D., \& Nelson, P. I. 1985, ApJ, 293, 192

Flaccomio, E., Micela, G., \& Sciortino, S. 2003, A\&A, 402, 277

Forbrich, J., Osten, R. A., \& Wolk, S. J. 2011, ApJ, 736, 25

Freeman, P. E., Kashyap, V., Rosner, R., \& Lamb, D. Q. 2002, ApJS, 138, 185

Getman, K. V., Flaccomio, E., Broos, P. S., et al. 2005, ApJS, 160, 319

Getman, K. V., Feigelson, E. D., Broos, P. S., Townsley, L. K., \& Garmire, G. P. 2010, ApJ, 708, 1760

Gregory, S. G., Wood, K., \& Jardine, M. 2007, MNRAS, 379, L35

Grosso, N., Briggs, K. R., Güdel, M., et al. 2007, A\&A, 468, 391

Güdel, M., Briggs, K. R., Arzner, K., et al. 2007, A\&A, 468, 353
Hartmann, L., Megeath, S. T., Allen, L., et al. 2005, ApJ, 629, 881

Herbig, G. H. 1954, PASP, 66, 19

Herbig, G. H. 1998, ApJ, 497, 736

Hillenbrand, L. A. 1997, AJ, 113, 1733

Jardine, M., Cameron, A. C., Donati, J.-F., Gregory, S. G., \& Wood, K. 2006, MNRAS, 367, 917

Kraft, R. P., Burrows, D. N., \& Nousek, J. A. 1991, ApJ, 374, 344

Lada, E. A., \& Lada, C. J. 1995, AJ, 109, 1682

Lada, E. A., Lada, C. J., \& Muench, A. 1998, in The Stellar Initial Mass Function (38th Herstmonceux Conference), ed. G. Gilmore, \& D. Howell, ASP Conf. Ser., 142,107

Lada, C. J., Muench, A. A., Luhman, K. L., et al. 2006, AJ, 131, 1574

Lee, N., Williams, J. P., \& Cieza, L. A. 2011, ApJ, 736, 135

Luhman, K. L. 1999, ApJ, 525, 466

Luhman, K. L., \& Rieke, G. H. 1999, ApJ, 525, 440

Luhman, K. L., Rieke, G. H., Lada, C. J., \& Lada, E. A. 1998, ApJ, 508, 347

Luhman, K. L., Stauffer, J. R., Muench, A. A., et al. 2003, ApJ, 593, 1093

Luhman, K. L., Lada, C. J., Hartmann, L., et al. 2005a, ApJ, 631, L69

Luhman, K. L., Lada, E. A., Muench, A. A., \& Elston, R. J. 2005b, ApJ, 618, 810

McCaughrean, M. J., Rayner, J. T., \& Zinnecker, H. 1994, ApJ, 436, L189

Megeath, S. T., Allen, L. E., Gutermuth, R. A., et al. 2004, ApJS, 154, 367

Mohanty, S., Jayawardhana, R., \& Basri, G. 2005, ApJ, 626, 498

Morales-Calderón, M., Stauffer, J. R., Hillenbrand, L. A., et al. 2011, ApJ, 733, 50

Muench, A. A., Lada, E. A., Lada, C. J., et al. 2003, AJ, 125, 2029

Muench, A. A., Lada, C. J., Luhman, K. L., Muzerolle, J., \& Young, E. 2007, AJ, 134, 411

Muzerolle, J., Hillenbrand, L., Calvet, N., Briceño, C., \& Hartmann, L. 2003, ApJ, 592, 266

Najita, J. R., Tiede, G. P., \& Carr, J. S. 2000, ApJ, 541, 977

Natta, A., Testi, L., \& Randich, S. 2006, A\&A, 452, 245

Preibisch, T. 2003, A\&A, 401, 543

Preibisch, T., \& Zinnecker, H. 2001, AJ, 122, 866

Preibisch, T., \& Zinnecker, H. 2002, AJ, 123, 1613

Preibisch, T., \& Zinnecker, H. 2004, A\&A, 422, 1001

Preibisch, T., Zinnecker, H., \& Herbig, G. H. 1996, A\&A, 310, 456

Preibisch, T., Kim, Y.-C., Favata, F., et al. 2005a, ApJS, 160, 401

Preibisch, T., McCaughrean, M. J., Grosso, N., et al. 2005b, ApJS, 160, 582

Siess, L., Dufour, E., \& Forestini, M. 2000, A\&A, 358, 593

Stelzer, B., \& Neuhäuser, R. 2001, A\&A, 377, 538

Stelzer, B., Flaccomio, E., Montmerle, T., et al. 2005, ApJS, 160, 557

Telleschi, A., Güdel, M., Briggs, K. R., Audard, M., \& Palla, F. 2007, A\&A, 468, 425

Townsley, L. K., Feigelson, E. D., Montmerle, T., et al. 2003, ApJ, 593, 874

White, R. J., \& Basri, G. 2003, ApJ, 582, 1109 